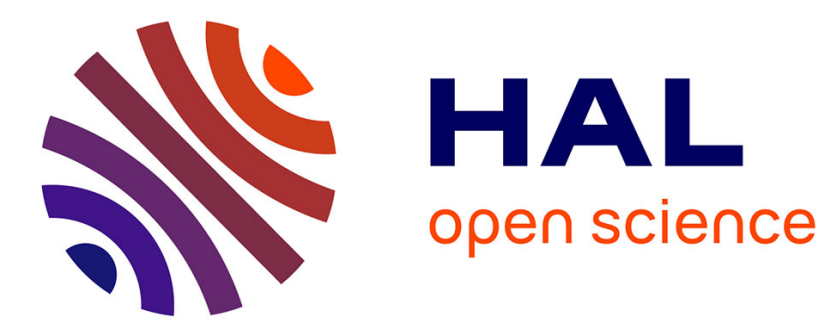

\title{
Les prêtres du Sarapieion C de Délos
}

Laurent Bricault

\section{To cite this version:}

Laurent Bricault. Les prêtres du Sarapieion C de Délos. Bulletin de Correspondance Hellenique, 1996, 120 (2), pp.597 - 616. 10.3406/bch.1996.4620 . hal-01817062

\section{HAL Id: hal-01817062 https://hal.science/hal-01817062}

Submitted on 16 Jun 2018

HAL is a multi-disciplinary open access archive for the deposit and dissemination of scientific research documents, whether they are published or not. The documents may come from teaching and research institutions in France or abroad, or from public or private research centers.
L'archive ouverte pluridisciplinaire HAL, est destinée au dépôt et à la diffusion de documents scientifiques de niveau recherche, publiés ou non, émanant des établissements d'enseignement et de recherche français ou étrangers, des laboratoires publics ou privés. 


\section{Laurent Bricault}

\section{Les prêtres du Sarapieion C de Délos}

In: Bulletin de correspondance hellénique. Volume 120, livraison 2, 1996. pp. 597-616.

Citer ce document / Cite this document :

Bricault Laurent. Les prêtres du Sarapieion C de Délos. In: Bulletin de correspondance hellénique. Volume 120, livraison 2, 1996. pp. 597-616.

doi : $10.3406 /$ bch.1996.4620

http://www.persee.fr/web/revues/home/prescript/article/bch_0007-4217_1996_num_120_2_4620 


\section{Abstract}

ID 2610 gives the list of Delian priests of Sarapis for the years 137/6-1 10/09 and shows that in this period there was a very strict rotation ofthe priesthood between the twelve tribes. Except for the years $109 / 8-106 / 5$, this rule seems to have been observed until at least $89 / 8$. After that date there was apparently no priest in charge of the Sarapeion, which was thenceforth entrusted to an ordinary zacoros. On the other hand, it is difficult to believe that the rule of rotation was respected between 167/6 and 140/39, unless it was subject to severe irregularities, which would be undemonstrable and inexplicable. Lastly we note that the priest Philocrates of Hamaxantia, the only official to serve twice as priest of Sarapis, held the office for the first time in 158/7 and again for a second term in 146/5.

\section{Résumé}

ID 2610 fournit la liste des prêtres déliens de Sarapis pour les années 137/6-110/09 et montre que, durant cette période, la prêtrise tourna entre les douze tribus de façon très rigoureuse. À l'exception des années 109/8-106/5, cette règle semble bien avoir été respectée au moins jusqu'en 89/8. Après cette date, il apparaît qu'aucun prêtre n'eut en charge le Sarapieion $\mathrm{C}$, désormais confié à un simple zacore. En revanche, il n'est pas possible de considérer que la règle de rotation fut respectée entre 167/6 et $140 / 39$, à moins de supposer quelques graves entorses à celle- ci, qui resteraient indémontrables et inexplicables. Notons enfin que le prêtre Philocratès d'Hamaxantia, le seul officiant connu jusqu'ici pour avoir occupé à deux reprises la charge de prêtre de Sarapis, fut en fonction pour la première fois en $158 / 7$ et pour la seconde fois douze ans plus tard en $146 / 5$.

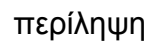

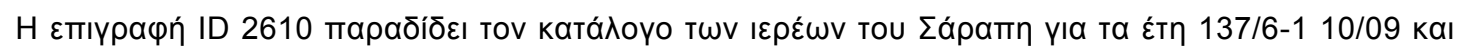

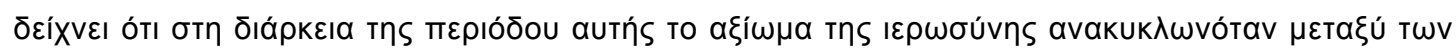

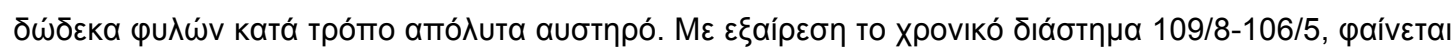

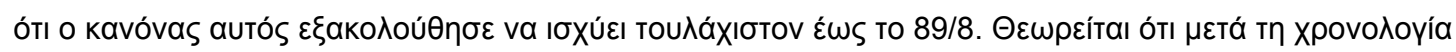

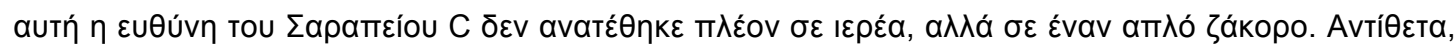

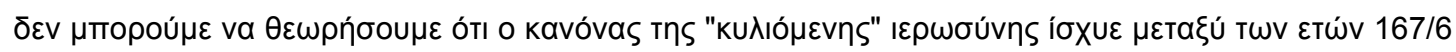

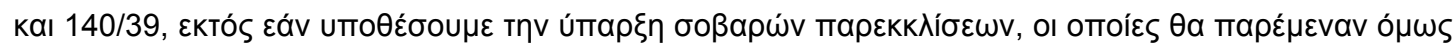

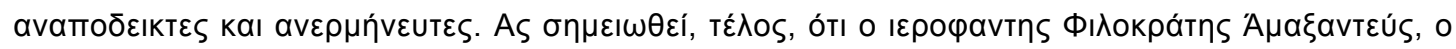

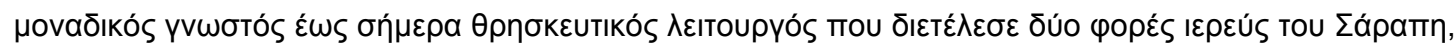

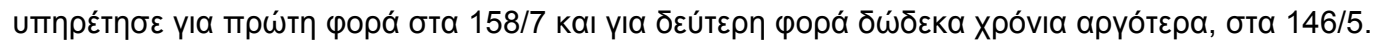

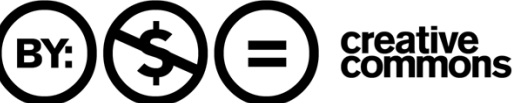




\section{Les prêtres du Sarapieion C de Délos*}

Un document fondamental pour la chronologie délienne à l'époque de la domination athénienne est la liste des prêtres officiels de Sarapis fournie par la stèle conservée au musée de Délos sous le $\mathrm{n}^{\circ}$ inv. $\Gamma 568$ et publiée en dernier lieu comme ID $2610^{1}$. Elle présente les noms des prêtres ayant officié entre $137 / 6$ et 110/09, les trois premières lignes conservées ne fournissant que quelques lettres sur lesquelles je reviendrai. Malheureusement, pour les années qui suivent, la situation est moins claire et la datation de la majorité des prêtrises est encore très incertaine.

Ayant eu l'occasion de retravailler les quelque 350 textes déliens concernant les cultes isiaques dans l'île à l'occasion de la préparation d'une nouvelle édition de la SIRIS de L. Vidman, j'ai repris, pour classer ces textes à peu près chronologiquement, le problème de la datation des prêtrises après $110 / 09$, et je crois être arrivé à cerner la réalité d'un peu plus près qu'elle ne le fut jusqu'à présent. Ce sont cette étude et ses résultats que je voudrais proposer ici.

La publication par Stephen V. Tracy, en 1982, de son étude sur IG II-III 2, $2336^{2}$ a permis de dater avec précision et certitude les sacerdoces de quatre des prêtres du Sarapieion C. Il s'agit des prêtres suivants :

\begin{tabular}{|c|c|}
\hline$\Delta \rho \alpha ́ \kappa \omega \nu \Phi \lambda v \varepsilon u ́ \varsigma$ & $103 / 2$ \\
\hline 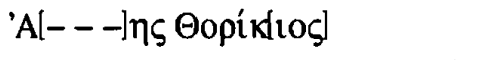 & $102 / 1$ \\
\hline 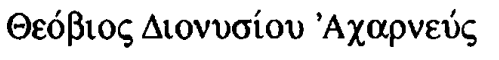 & $101 / 0$ \\
\hline 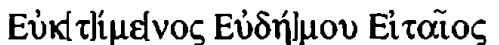 & $97 / 6$ \\
\hline
\end{tabular}

Comme on peut le constater, la datation de ces quatre prêtrises confirmerait a priori l'hypothèse de W. B. Dinsmoor ${ }^{3}$ selon laquelle la rotation des tribus ne s'est pas interrompue après le sacerdoce de Sosion en 110/09, même si cette date marque la fin d'un cycle, Sosion appartenant à la douzième tribu (dème Oivón), et si les années 109/8108/7 laissent apparaître une sérieuse entorse à la rigueur de cette rotation.

En publiant une inscription délienne inédite conservée au musée de Trévise", Margherita Guarducci permit de dater une nouvelle prêtrise, celle d"A

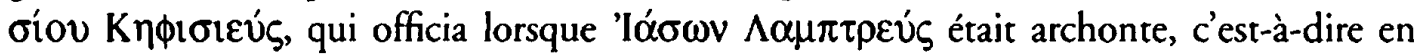
$109 / 8^{5}$. Durant son sacerdoce, Apollophanès fut assisté par son neveu $\Delta$ tovv́otos

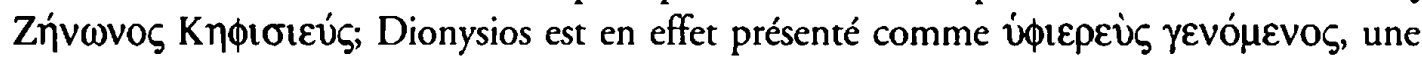


expression que l'on doit traduire par "devenu prêtre auxiliaire ", alors même que son oncle est en charge de son office ${ }^{7}$. Cela ne peut, me semble-t-il, s'expliquer que par une incapacité (partielle?, temporaire?, je ne sais) d'Apollophanès à assurer son service, cette situation nécessitant l'adjonction d'un assistant, ici son propre neveu ${ }^{8}$.

Constatant qu'il s'agit de la seule mention d'un úфtepev́s dans la documentation délienne, $M$. Guarducci s'est demandé si les années $137 / 6,120 / 19,116 / 5$ et 113/2, pour lesquelles la liste $I D 2610$ donne à chaque fois deux noms, pouvaient correspondre à un cas de figure semblable à celui de 109/8. Sa réponse, positive, ne me paraît pas s'imposer?.

Notons d'emblée que la présence de la conjonction ká, qui peut tout aussi bien signifier la simultanéité d'office que la succession des prêtrises, ne nous est ici d'aucun secours pour résoudre la difficulté.

A priori, rien n'indique non plus que ces quatre années présentent des situations comparables ; dans certains cas, il peut s'agir d'un prêtre et de son auxiliaire, dans d'autres d'un prêtre et de son remplaçant/successeur.

Les deux prêtres nommés pour l'année $137 / 6$ n'apparaissent dans aucun autre texte; il est impossible de choisir.

En 120/19, Damon, premier nommé dans la liste, est mentionné comme ípeús (CE $101=I D 2121)$, mais Téléphos n'apparaît nulle part ailleurs; impossible de choisir là non plus.

En 116/5, Stratodamos, premier nommé, n'est pas connu par ailleurs, mais Dionysios, lui, apparaît sur nombre de documents (CE 113-116 = ID 2057-2058, 2078 et

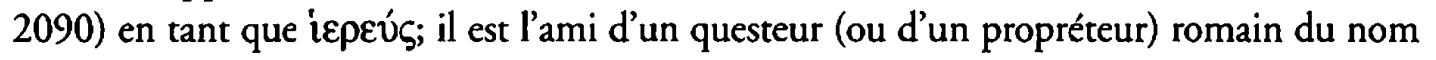
de Marcus (ID 1843), hoplophore des dieux du Cynthe en 119/8 (ID 1877), tandis que sa

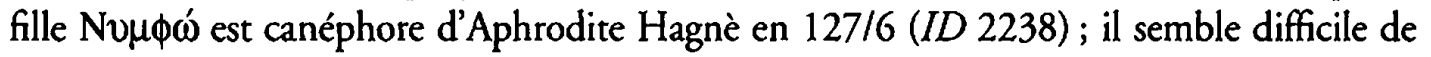
ne voir en lui qu'un auxiliaire et il faut peut-être considérer que Stratodamos est mort en charge et qu'il fut remplacé par Dionysos pour achever l'année dévolue à la sixième tribu.

Aucun des deux prêtres nommés pour $113 / 2$ n'est connu par ailleurs.

Globalement, les raisons précises pour lesquelles quatre années, entre 137/6 et 110/09, ont connu deux prêtres de Sarapis nous échappent encore, mais il est bien difficile de croire que l'on eût affaire à chaque fois à un prêtre et à son assistant.

Deux autres prêtrises ont une datation assurée grâce à la mention, sur un même document, du prêtre et de l'archonte athénien qui, dans les deux cas, peut être daté avec

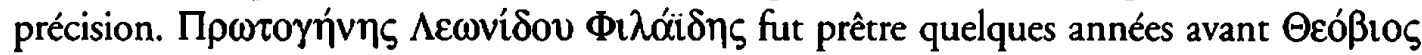
(dont le sacerdoce se place en 101/0), l'année de l'archontat d"A $\gamma \alpha \theta 0 \kappa \lambda \tilde{\eta} \varsigma$, c'est-à-dire en 


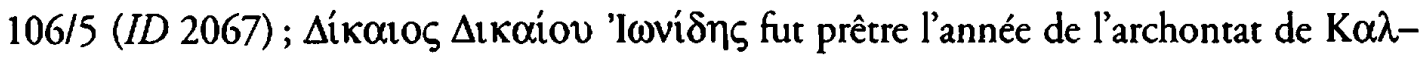
$\lambda$ ías, c'est-à-dire en $94 / 3$ (ID 2081).

Sept prêtrises (et un auxiliariat) ont donc une date assurée, ce qui nous donne l'embryon de liste suivant :

\begin{tabular}{|c|c|}
\hline 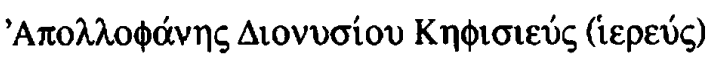 & $(109 / 8)$ \\
\hline 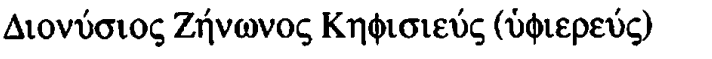 & $(109 / 8)$ \\
\hline 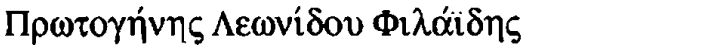 & $(106 / 5)$ \\
\hline$\Delta \rho \alpha ́ \kappa \omega v \Phi \lambda v \varepsilon v ́ \varsigma$ & $(103 / 2)$ \\
\hline 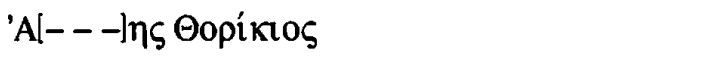 & $(102 / 1)$ \\
\hline 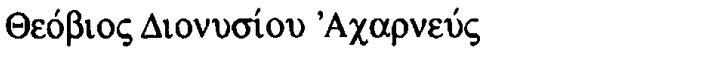 & $(101 / 0)$ \\
\hline 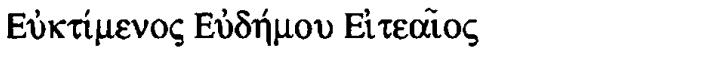 & $(97 / 6)$ \\
\hline 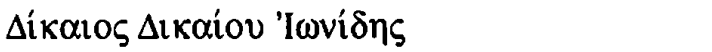 & $(94 / 3)$ \\
\hline
\end{tabular}

Hormis pour la période 108/7-107/6 qui présente une lacune de deux ans entre le sacerdoce d'un prêtre issu de la première tribu et celui d'un prêtre issu de la deuxième, la rotation des tribus selon l'ordre officiel semble parfaitement se poursuivre, au moins jusqu'en $94 / 3$, et pourquoi pas encore quelque temps après.

C'est ici que les certitudes laissent la place aux hypothèses. Mais nous allons voir que la situation est moins désespérée qu'il ne semble.

Les textes déliens nous font connaître les prêtres suivants ayant exercé leur sacerdoce après 110/09, si l'on s'en tient aux analyses de P. Roussel, suivies par presque tous depuis $^{10}$. Pour des raisons de commodité, je classerai dans un premier temps ces prêtres selon l'ordre officiel des tribus auxquelles ils appartiennent.

\begin{tabular}{|c|c|}
\hline 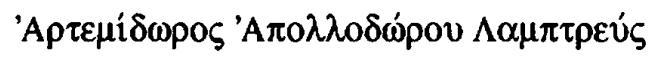 & I \\
\hline 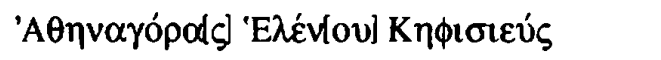 & I \\
\hline$[---' A] \lambda \alpha \imath \varepsilon v ́ s ?$ & II \\
\hline 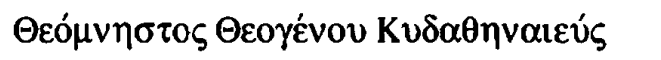 & III \\
\hline 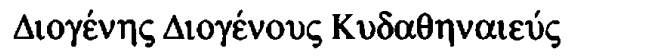 & III \\
\hline 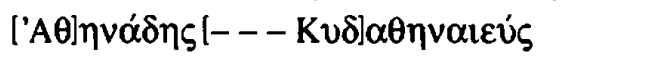 & III \\
\hline$[---\Pi \alpha \mathrm{u}] \alpha v \imath \varepsilon \dot{\zeta} \zeta$ & III \\
\hline 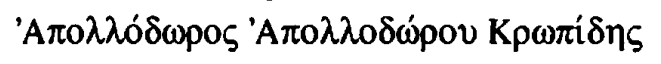 & IV \\
\hline 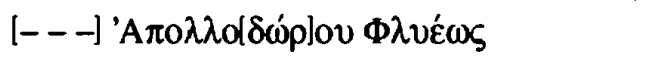 & V \\
\hline 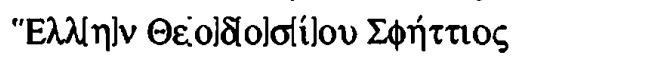 & VI \\
\hline 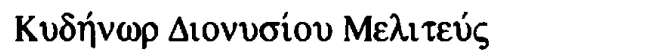 & VIII \\
\hline
\end{tabular}




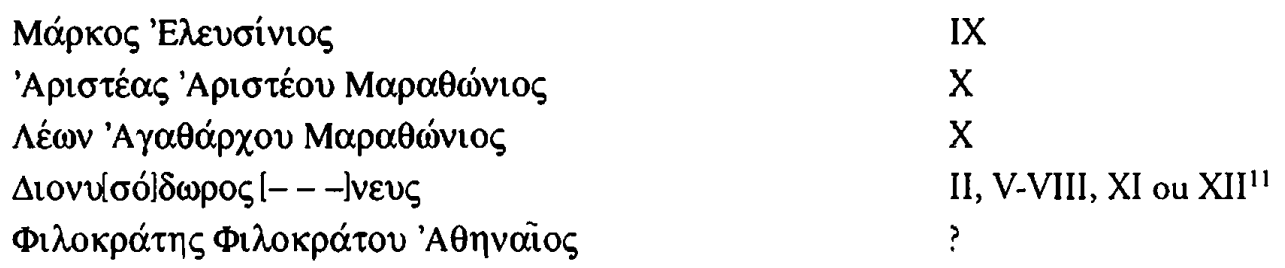

Considérant comme acquis que l'ordre de rotation des tribus s'est bien perpétué après 110/09 (sauf peut-être durant les années 108/7-107/6) on peut envisager les cas de figure suivants, sans descendre trop bas, la documentation faisant défaut après le saccage de l'île par les troupes de Mithridate:

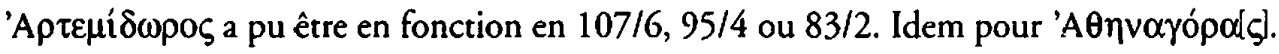

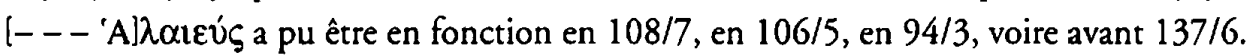

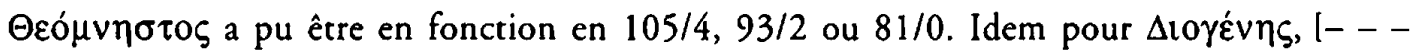

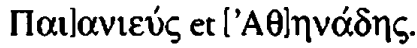

'A $\pi 0 \lambda \lambda o ́ \delta \omega \rho o \varsigma$ a pu être en fonction en 104/3 ou 92/1.

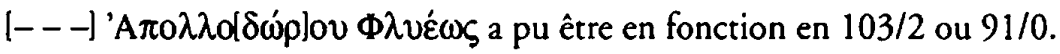

"E $\lambda \lambda \lambda \eta l v$ a pu être en fonction en $102 / 1$ ou 90/89.

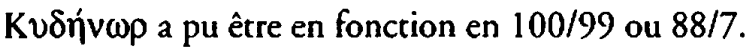

Mápкo $\zeta$ a pu être en fonction en $99 / 8$ ou $87 / 6$.

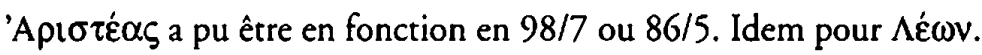

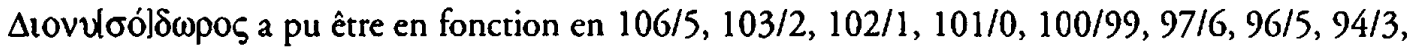
$91 / 0,90 / 89,89 / 8,88 / 7,85 / 4$ ou $84 / 3$.

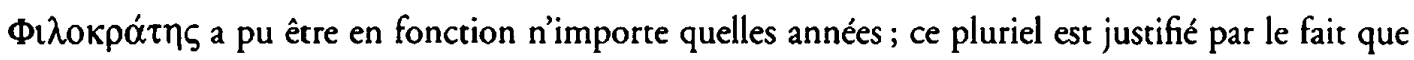
deux textes (ID 2068-2069) indiquent qu'il est prêtre (de Sarapis) pour la seconde fois.

Voyons maintenant les informations que nous donnent les textes pour essayer de clarifier un peu tout cela.

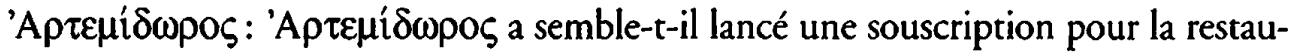

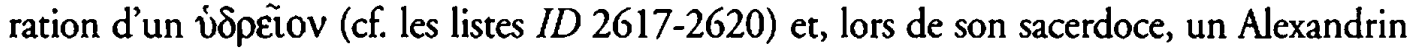
du nom de Démétrios a fait la consécration d'un dromos, de sphinges et d'une horloge (ID 2087-88). Le zacore d"A (cf. infra) et comme le zacore qui est en poste pour la dix-huitième année lors de l'épimélétat de Callimachos ca 70-60: il peut s'agir d'une, de deux ou de trois personnes différentes.

S'il ne s'agit pas des mêmes personnes, tout rapprochement devient caduc et aucune précision chronologique ne peut en être tirée.

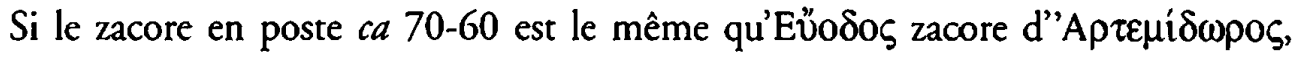
deux possibilités s'offrent à nous. En effet, s'il prend la peine $c a$ 70-60 de préciser " zacore pour la dix-huitième année ", c'est pour dater les deux textes (ID 2161 et 2205) dans lesquels aucun prêtre n'est mentionné; juste après le nom de l'épimélète, on lit celui du 
zacore, une pratique unique en son genre. Ces dix-huit années sont donc consécutives, sinon une telle indication n'aurait aucune utilité. Dans ce cas, l'année où il fut zacore sous

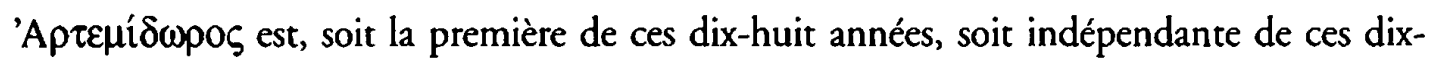

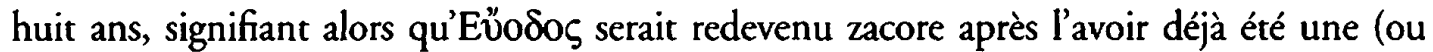

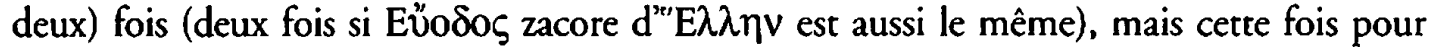
pallier, semble-t-il, l'absence de prêtre. Si l'on adopte la première hypothèse, 'A $\delta \omega \rho o \zeta$ est le dernier prêtre de la liste ayant eu un zacore et il se place après $\Delta$ íkolos (prêtre en 94/3) qui eut pour zacore son propre frère 'A $\pi 0 \lambda \lambda \omega$ vios (ID 2081, 2094,

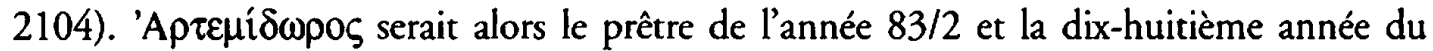

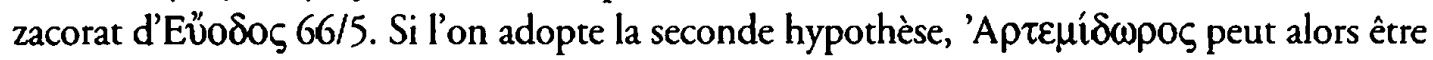
le prêtre de $95 / 4$ comme celui de $83 / 2$.

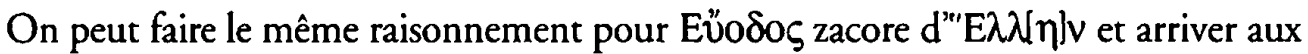
conclusions suivantes. Si son zacorat sous "E $\lambda \lambda[\eta] v$ est le premier des dix-huit, "E $\lambda \lambda\langle\eta \eta\rangle$ est le prêtre de 78/7, s'il est indépendant de ces dix-huit années, "E $\lambda \lambda\lceil\eta\rceil v$ doit être le prêtre de 90/89, car une prêtrise en 78/7 situerait $I D 2161$ et 2205 après 60 , à une date trop tardive pour placer l'épimélétat de $\Delta \eta \mu \varepsilon ́ \alpha$.

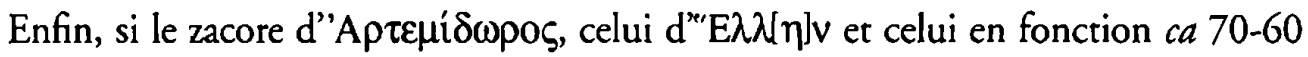
sont un seul et même personnage, cela laisse le champ ouvert à toute supposition chronologique, mais nous apprend qu'un zacore a pu être deux fois non consécutives en poste, et sans doute y montrer suffisamment de qualités pour qu'on lui confie la garde du sanctuaire lorsque celui-ci n'aura plus de prêtre pour l'administrer.

Notons en outre que les noms de plusieurs des souscripteurs mentionnés dans $I D$ 2617-2620 laissaient à penser à P. Roussel que ces listes n'étaient pas antérieures au Ir $\mathrm{s}$. Je

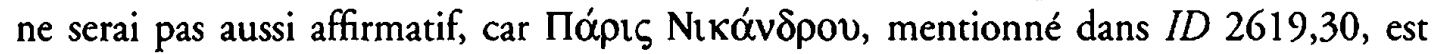
connu par deux dédicaces (ID 2122 et 2168 ) datées respectivement de $118 / 7$ et $112 / 1$; toutefois, on peut admettre qu'il souscrivit longtemps après avoir dédié, ou bien qu'il est

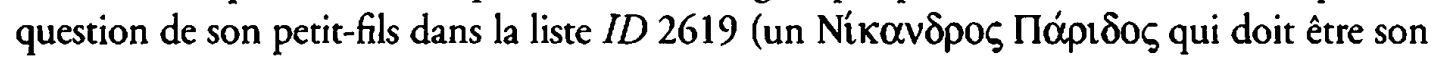
fils est mentionné dans ID 2622 a II,33).

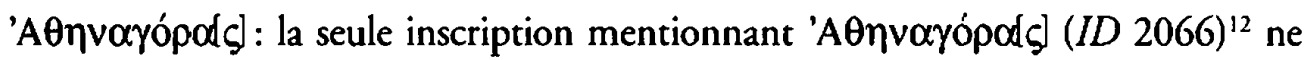

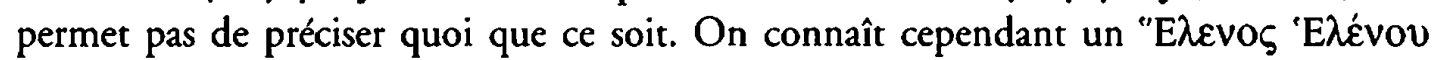

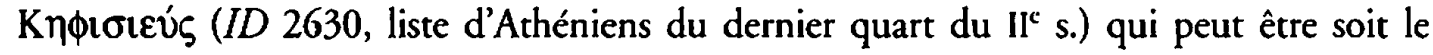
frère, soit le père d"A $A \eta v \alpha \gamma o ́ p \alpha \zeta$ l. La date de 83/2 peut apparaître alors comme un peu

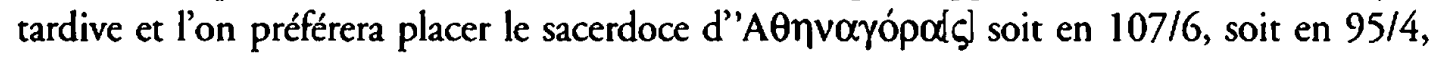
sans pouvoir choisir de manière définitive entre l'une et l'autre date.

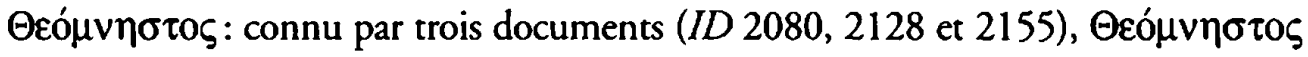
devient prêtre d'Artémis en l'île en 102/1 (IG II/III 2, 2336,79). Cette prêtrise, sans aucun doute plus importante et prestigieuse que celle de Sarapis, peut apparaître comme une promotion pour ce prêtre et a dû être assumée plus tard. Le dédicant d'ID 2155, Sporius, un 
Romain, est connu par plusieurs autres textes (ID 2156, 2378-79, 2446, 2449 et peut-être également 1687), le premier de ceux-ci étant daté avec certitude de 103/2. Cela invite à

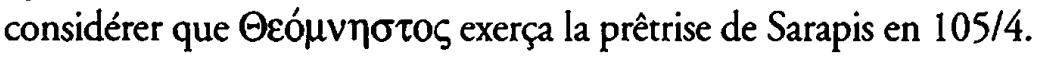

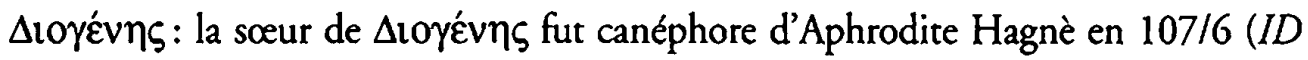
2232). On a songé à voir en lui l'un des thesmothètes de 97/6 (IG II/III 2, 2236,249), mais le texte de S. V. Tracy semble infirmer cette proposition, la lacune (environ 4 lettres)

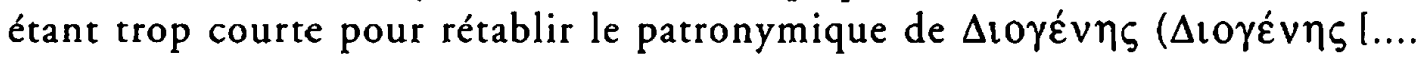

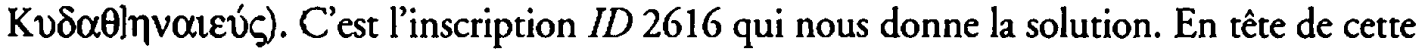

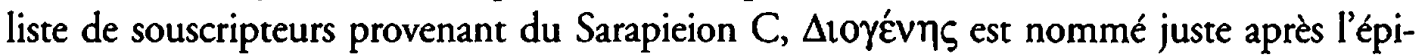
mélète de l'emporion, comme lui avec femme et enfants. S'ensuivent les noms des simples souscripteurs, pour la majorité des Romains (ce qui invite sans doute déjà à éliminer la date de 81/0). Or cette souscription eut lieu alors que l'épimélète de l'île était "Ap[- - -]. Au début du ${ }^{\text {er }}$ s., trois épimélètes dont le nom commence par ces deux lettres sont

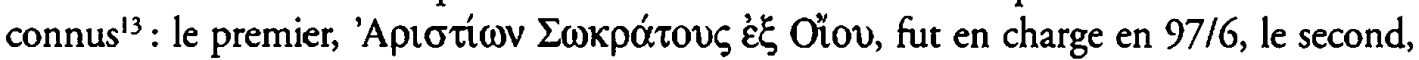

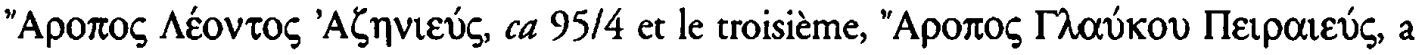

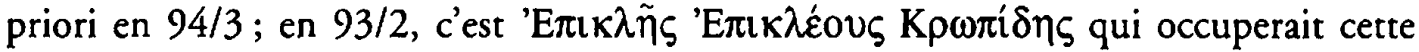
charge selon une datation communément admise. Si la date de l'épimélétat d'Aristion est assurée par $I G \mathrm{II} / \mathrm{III} 2,2336,229$, les trois autres ne le sont peut-être pas autant. Celle de

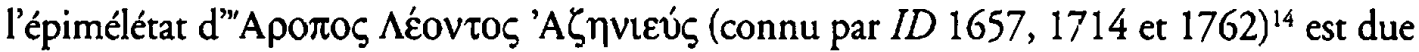
à une conjecture de Dinsmoor ${ }^{15}$ qui reste d'ailleurs prudent sur la solidité de cette data-

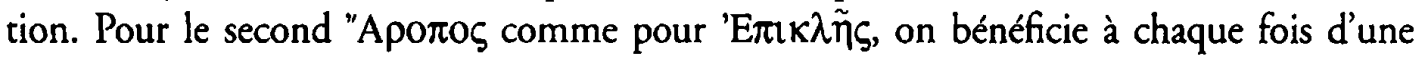

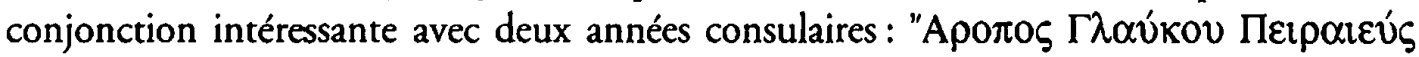
est épimélète l'année où $\mathrm{C}$. Coelius et L. Domitius sont consuls, c'est-à-dire en 94 (ID

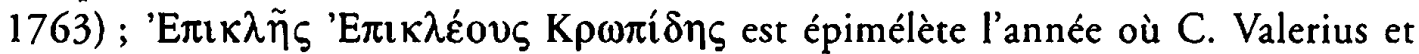
$M$. Herennius sont consuls, c'est-à-dire en 93 (ID 1764). Cela n'implique pas cependant

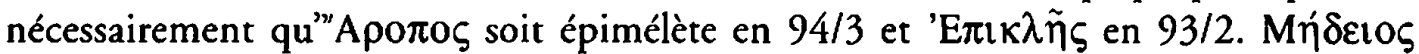

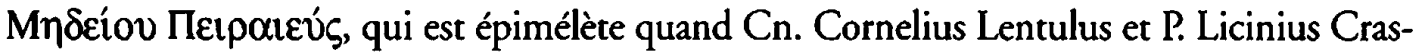
sus sont consuls (en 97; ID 1757), est en poste en $98 / 7$ et non en $97 / 6$ (IG II/III 2, 2336,189). Je croirais plus volontiers que l'épimélète de $95 / 4$ est "Аро

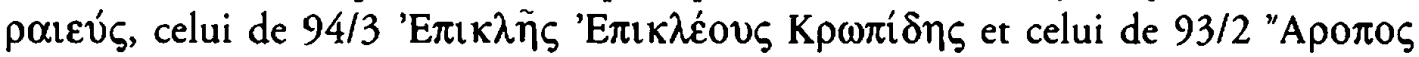

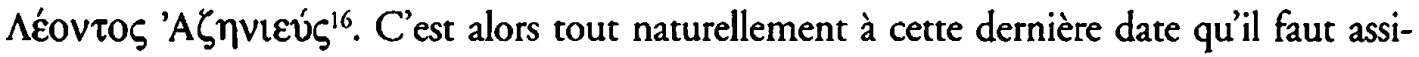
gner la prêtrise de $\Delta$ lo $\gamma^{\prime} v \eta \varsigma^{17}$.

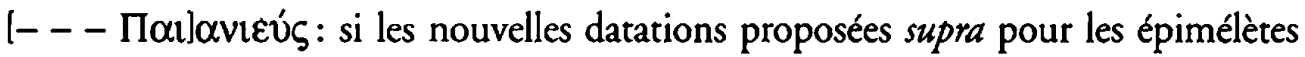
de l'île des années 95/4-93/2 sont correctes, ce prêtre, connu par ID 2039 et 2159, fut en

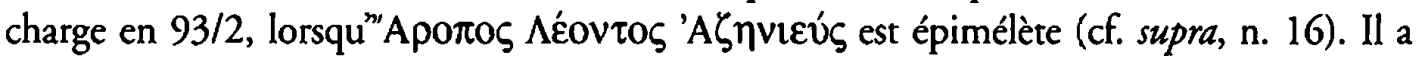
pu être le prédécesseur, le successeur ou l'assistant de $\Delta$ to $\gamma^{\prime} v \eta \zeta$, tous deux étant de la troisième tribu.

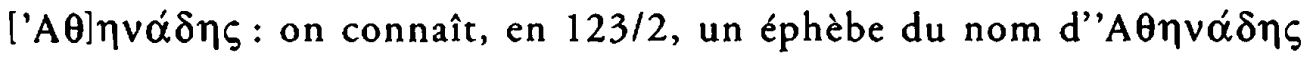

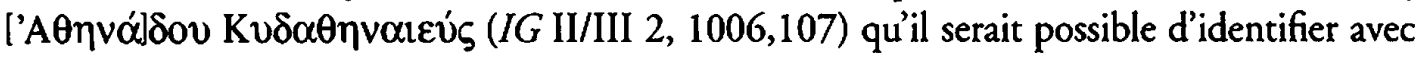
le prêtre de Sarapis. Par ailleurs, l'artisan auteur d'ID 2157 (seule inscription mentionnant 


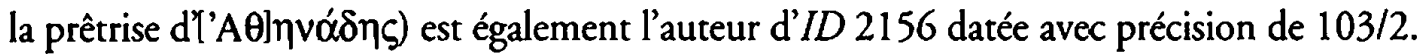
Selon toute vraisemblance, il faut éliminer la date de 81/0. Mais si l'on place la prêtrise

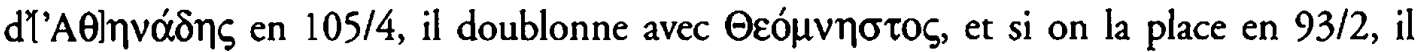

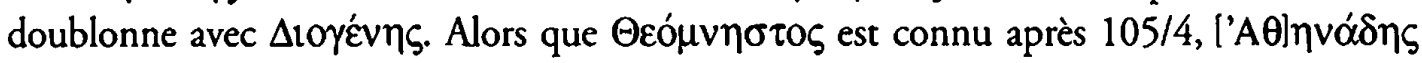
ne l'est pas, ce qui peut inciter à supposer qu'il mourut l'année de sa prêtrise et fut rem-

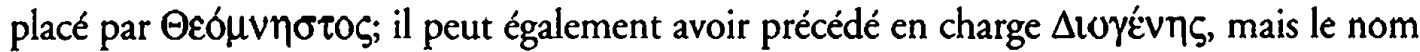
de l'artisan invite à préférer la première solution, sans pour autant exclure la seconde.

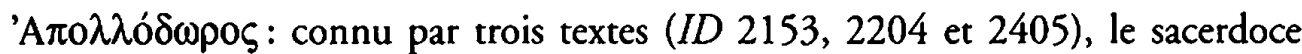
d"A raît que dans les trois mêmes textes; le cleidouque nommé dans ID 2204 et 2405 fait une dédicace en $98 / 7$ pour le nouvel épimélète (ID 1816) ; quant au dédicant d'ID 2153, il se retrouve peut-être dans le catalogue ID 2595,22-23, sans que cela soit déterminant pour notre problème. L'incertitude reste donc entière entre $104 / 3$ et $92 / 1$.

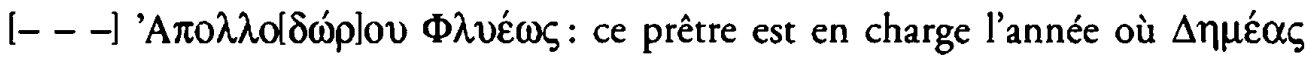

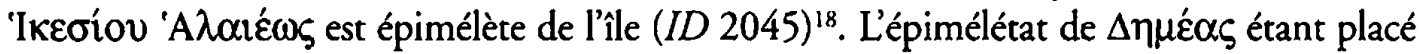
ca 90/89, la prêtrise du fils d'Apollodoros est à dater de $91 / 0$ plutôt que de 103/2' .

"E $\lambda \lambda\lceil\eta] v$ : d'abord rattachée au culte syrien par P. Roussel (BCH 38 [1908], p. 387, 389), la prêtrise d"E$\lambda \lambda \lambda \eta\rceil v$ fut attribuée au culte isiaque dans ses $C E$ puis dans le com-

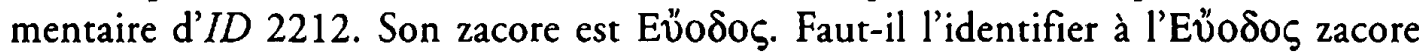

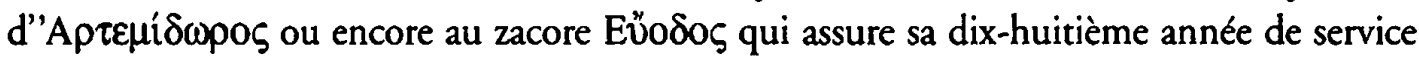
ca 70-60 ? Il y'a là trop d'incertitudes pour pouvoir décider avec assurance (cf. supra). Le prêtre officiant en 102/1 étant connu, il est envisageable de dater la prêtrise $\mathrm{d}^{\prime \prime} E \lambda \lambda[\eta] v$ de 90/89 plutôt que de $78 / 7^{20}$.

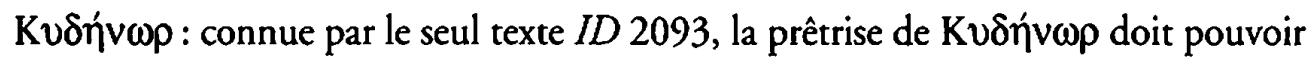

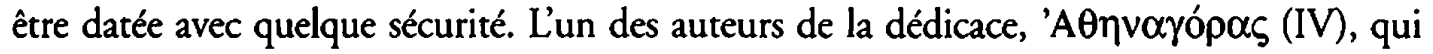
appartient à une famille bien connue (cf. ID 1871, 1891 et 2092), fut éphèbe en 106/5; dans le texte de la dédicace qu'il fit déposer dans le sanctuaire lors de la prêtrise de

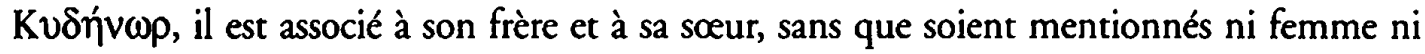
enfants; la dédicace est pour son oncle; il semble difficile de placer celle-ci en $88 / 7$, dix-

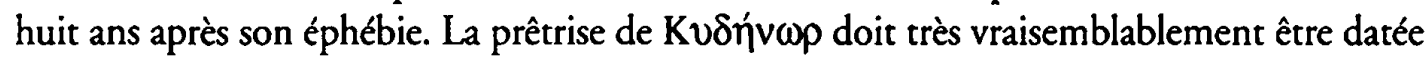
de $100 / 99$.

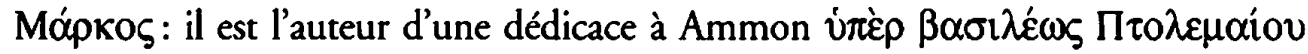

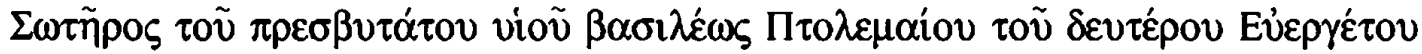
(ID 2037). Il s'agit bien sûr de Ptolémée IX Sôter II ${ }^{21}$, fils de Ptolémée VIII Évergète II et de Cléopâtre III, qui régna sur l'Égypte conjointement avec sa mère de $117 / 6$ à $107 / 6$ avant d'être écarté du trône au profit de son cadet Ptolémée X Alexandre ${ }^{\text {Ir }}$ et de revenir au pouvoir de $88 / 7$ à $81 / 0$. De $107 / 6$ à $88 / 7$ il règne à Chypre, en exil. P. Roussel considérait que la dédicace ne pouvait être rapportée qu'à une date postérieure au retour du souve- 
rain en Égypte, c'est-à-dire entre 88 et 81 . M.-Fr. Baslez ${ }^{22}$ allait plus loin en envisageant que cette dédicace à Ammon, fait assez rare il est vrai, devait être liée à la reprise de Thèbes, la cité d'Ammon, par Ptolémée Sôter II en 86/5. Cela me semble extrêmement peu convaincant. Si Mópкoৎ a indiqué que Sôter II était le fils aîné d'Évergète II, c'est évidemment pour marquer sa prééminence naturelle sur Alexandre $\mathrm{I}^{\text {er }}$. Et à quel moment un prêtre de Sarapis, qui dut bénéficier de la générosité de Sôter II, pouvait-il flatter le mieux celui qu'il voulait honorer en rappelant son état d'aîné et sa légitimité sinon lorsque celuici se trouvait en concurrence avec son cadet, puis dépossédé de son trône par celui-ci, c'està-dire avant $88^{23}$ ? La dédicace à Ammon, dont la popularité dans l'Égypte des Égyptiens était infiniment plus grande que celle de Sarapis dont on sait le peu de succès qu'il rencontra auprès des indigènes, va peut-être également dans ce sens de l'affirmation d'une légitimité24. Il faut noter par ailleurs que le sacerdoce de Mópкоৎ doit être assez proche (le précédent ?) de celui d"Apı $\sigma \tau \varepsilon_{\alpha} \alpha$ puisque dans les deux cas, ce qui est unique me semble-t-il,

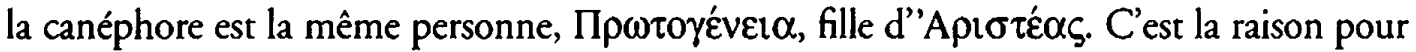
laquelle je daterais la prêtrise de Mópкos de 99/8 plutôt que de $87 / 6$.

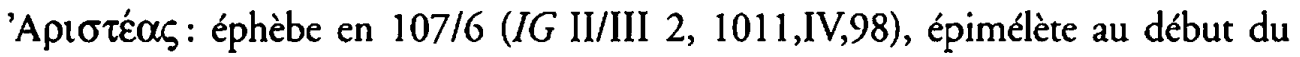

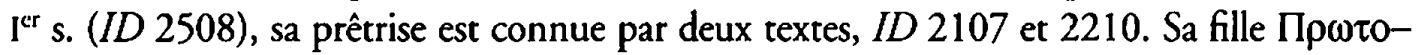
$\gamma \varepsilon ́ v \varepsilon ı \alpha$, canéphore lors de son sacerdoce avec sa sœur $\Phi^{\prime} \lambda \alpha$, l'est également sous le sacer-

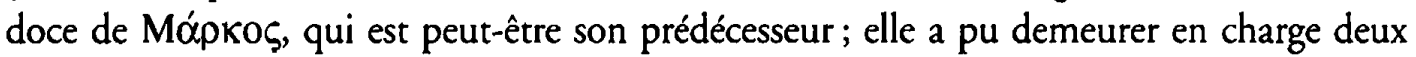
années de suite, et être assistée la deuxième année par sa sœur alors que leur père est en fonction. ID 2107 indique qu'A quelque chose consacré lors du sacerdoce du premier (bâtiment, statue... ?) a fait l'objet d'une restauration lors du sacerdoce du second. Pour toutes ces raisons, je serais enclin à

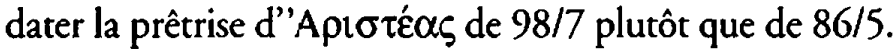

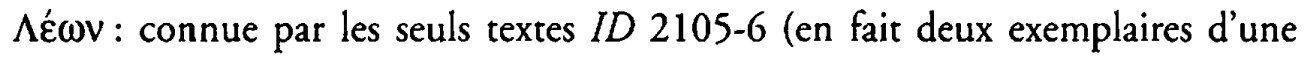
même dédicace), la prêtrise de $\Lambda \varepsilon ́ \omega v$ est a priori difficilement datable avec précision. Le dédicant, Quintus, fils de Caius, doit être le fils ou le frère du prêtre de Sarapis de 115/4; un prêtre des Dioscures Cabires du même nom est connu (ID 1905,1). La dédicace est faite sur ordre du dieu transmis par l'onéirocrite, signe que le personnel du sanctuaire est assez étoffé, ce qui surprendrait pour une date postérieure à $88 / 7$. La prêtrise de $\Lambda \varepsilon ́ \omega v$ doit dater de $98 / 7$ plutôt que de $86 / 5$, comme celle d'Apı $\sigma \tau \varepsilon ́ \alpha \varsigma$, qui a pu le précéder en

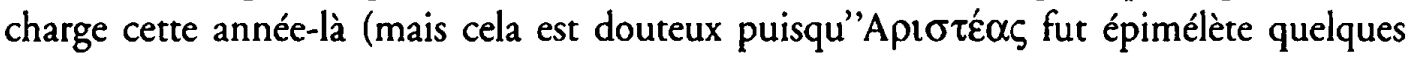
années plus tard) ou bien lui succéder. On pourrait concevoir que, $\Lambda \dot{\varepsilon} \omega v$ mort en charge,

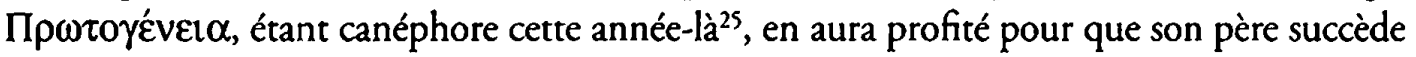
au prêtre défunt, par on ne sait quel moyen.

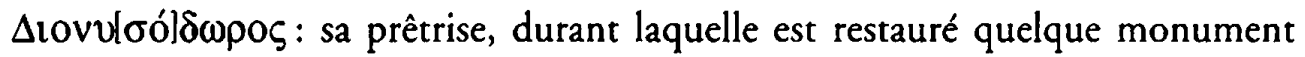

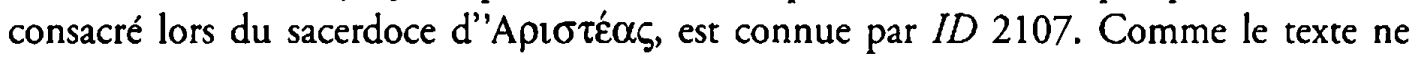

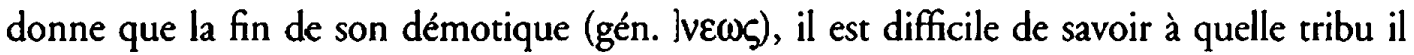
appartenait (cf. supra). Si l'on accepte de dater la prêtrise d"A

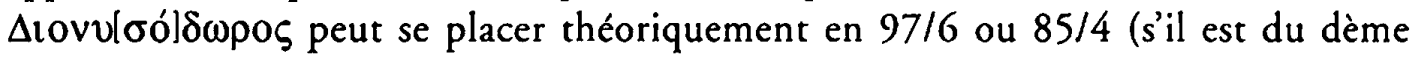




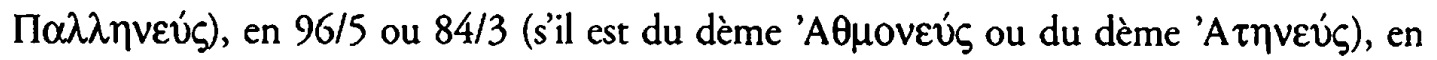

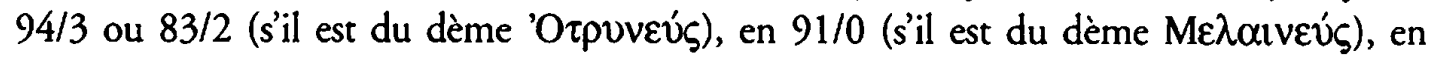

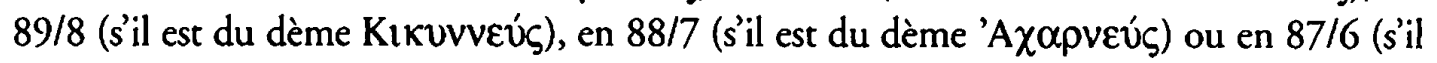
est du dème $A i \xi \omega v \varepsilon v ́()$. Comme il est improbable que la cause des dégâts occasionnant cette restauration soit le temps, il doit s'agir d'une catastrophe, humaine ou naturelle; P. Roussel songeait au saccage pontique, mais la raison peut être autre. Quoi qu'il en soit, les dégâts ont pu être causés aussi bien quelques jours ou quelques semaines après la consécration que dix ans plus tard; aucune date ne peut donc être exclue sur ce critère d'une trop grande proximité chronologique avec la date de consécration ${ }^{26}$. D'après les recoupements énoncés dans les pages précédentes, aux seules années $96 / 5,89 / 8$ et $88 / 7$ n'ont pas été attribués de prêtres; c'est peut-être l'une de ces années-là qu'il faut placer la prêtrise de

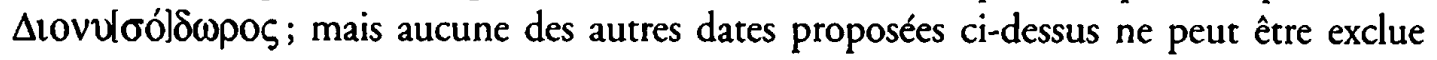
absolument.

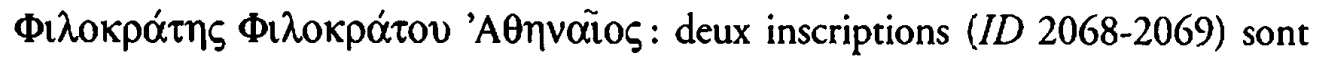

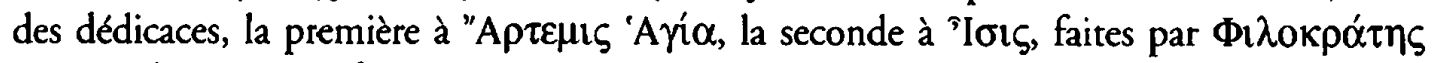

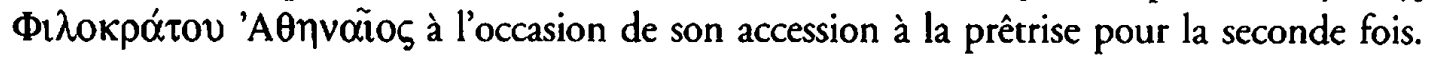

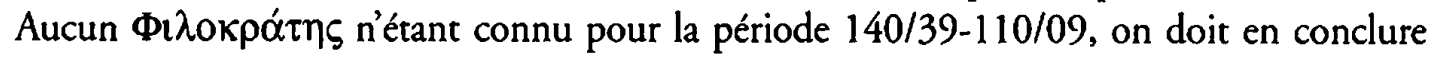
qu'il exerça ses deux prêtrises soit avant 140/39, soit après 110/09 ou bien encore l'une avant et l'autre après.

La liste de prêtres et de pompostoles ID 2605, datée précisément de 158/7,

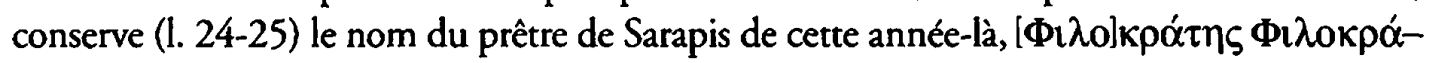

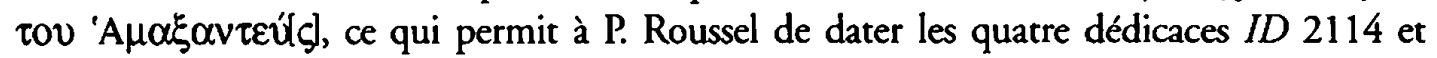

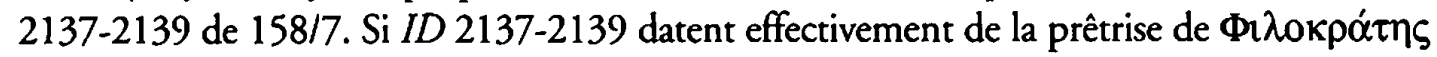
d'Hamaxantia, il n'en va cependant peut-être pas de même pour ID 2114. La dédicace est

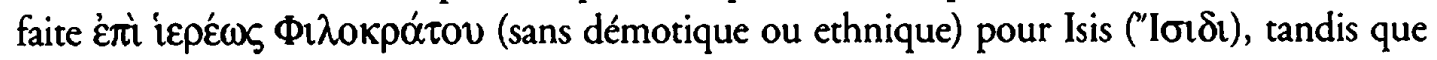

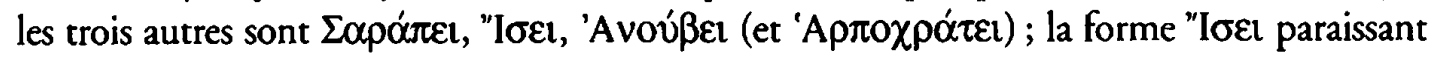

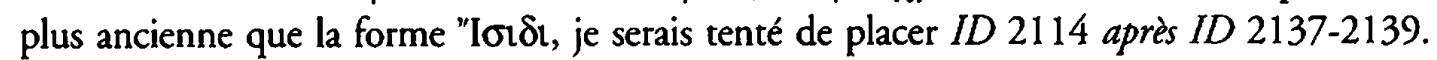

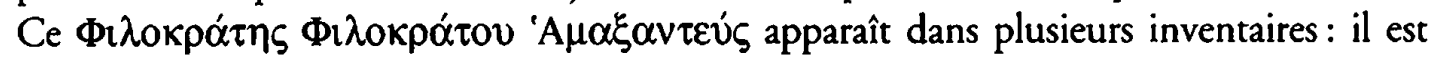
garant dans l'inventaire d'Anthestérios (ID 1416 B II,27-28) (157/6); des dons, effectués sous son sacerdoce sont enregistrés dans l'inventaire de Callistratos (156/5) (ID $1417 \mathrm{~B}$,

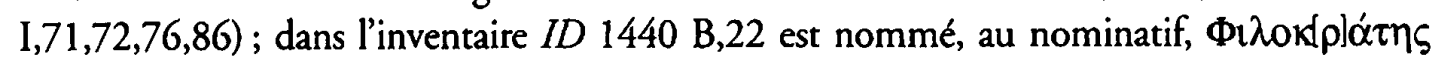

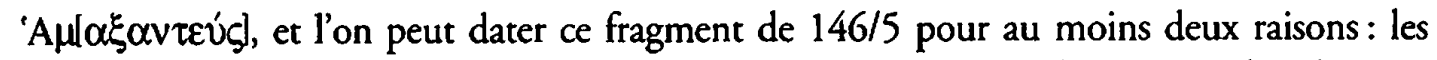
offrandes mentionnées dans ce fragment se retrouvent toutes, dans le même ordre, dans $I D$ 1442 B,42-54 (145/4), et la transmission (ID $1440 \mathrm{~B}, 10-11$ ) faite l'année de l'établissement de l'inventaire a disparu dans $I D 1442$ où les offrandes sont reportées sans interruption; dans l'inventaire de Métrophanès (145/4) (ID $1442 \mathrm{~A}, 57)$ sont enregistrées des offrandes faites lors du sacerdoce de $\Phi 1 \lambda$ okpótm (correspondant aux dédicaces ID 21382139) qui "n'ont dû être enregistrées qu'avec retard " (comm. à ID 1442, p. 151); enfin, il est mentionné comme prêtre dans l'inventaire ID 1415,7, que l'on peut très difficilement dater de 158/7 comme le pensaient P. Roussel et $M$. Launey sur la seule mention du nom de Philocratès; en effet, aucune des offrandes mentionnées dans ce fragment n'apparaît ni 
dans $I D 1416$ (157/6), ni dans $I D 1417$ (156/5), ni même dans $I D 1442$ (145/4); le seul point commun est la mention d'une donatrice nommée Bérénice. Mais, même s'il s'agit de la même personne (la nature de son offrande a disparu d'ID 1415), sa donation a pu être conservée depuis avant $157 / 6$ jusqu'à la date de composition de l'inventaire $I D 1415$, que, pour toutes ces raisons, je serais enclin à placer après 145/4, c'est-à-dire après la seconde prêtrise de Philocratès d'Hamaxantia.

La question qui se pose est de savoir si l'on a affaire à un seul ou à deux prêtres différents. En suivant le raisonnement présenté par M.-Fr. Baslez dans plusieurs études ${ }^{27}$, on pourrait imaginer que Philocratès, fils de Philocratès, Athénien, est le jeune fils de Philocratès, fils de Philocratès, d'Hamaxantia et d'une femme de naissance étrangère ${ }^{28}$. Cela signifierait que le père comme le fils ont tous deux été prêtres de Sarapis à deux reprises : le père en 158/7 (indiscutable) et $146 / 5$ (plus que probable), le fils deux fois (cf. ID 20682069) avant $141 / 0$ ou une fois avant $141 / 0$ et une fois après $109 / 8$ (en $99 / 8$ puisqu'il appartient à la neuvième tribu), voire deux fois après 109/8. Cette dernière hypothèse ne peut être retenue puisqu'il ne s'est pas écoulé suffisamment de temps entre $109 / 8$ et $89 / 8$ pour que la neuvième tribu se soit trouvée deux fois en situation de voir le prêtre de Sarapis choisi dans ses rangs. Il est par ailleurs peu probable que Philocratès Athénien, désormais assez âgé, ait tenu la prêtrise de Sarapis pour la deuxième fois en 99/8, c'est-à-dire au minimum 42 ans après sa première prêtrise. Seule la première hypothèse demeure donc

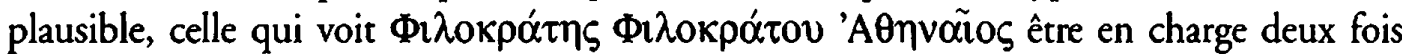
avant 141/0. Mais le fait qu'il n'apparaisse jamais dans les inventaires et que ses deux prêtrises, ajoutées à celles de son père, mettent encore plus à mal une rotation qui semblait être à peu près respectée entre 158/7 et 146/5 (dates des deux prêtrises paternelles) invite à considérer cette hypothèse avec beaucoup de circonspection.

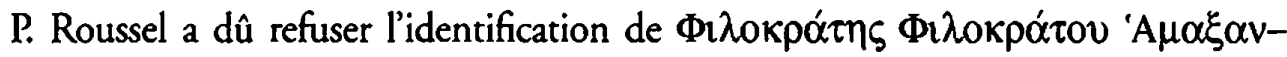

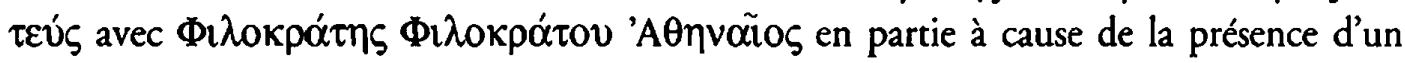
démotique chez le premier et d'un ethnique chez le second, encore qu'ils ne soient évi-

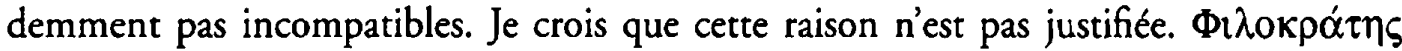

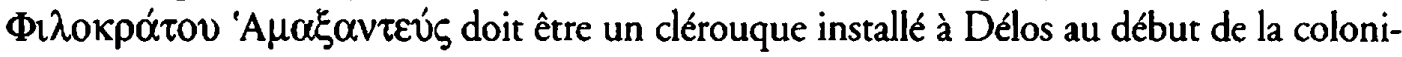
sation athénienne. Il porte alors son démotique et devient prêtre de Sarapis en 158/7. Stabilisé à Délos, il reste attaché aux cultes isiaques puisqu'il semble être mentionné comme donateur peu avant 146/5. Il abandonne peu après son démotique pour un ethnique,

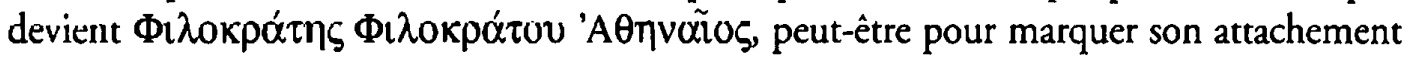
à Délos, peut-être à l'occasion de sa seconde accession à la prêtrise de Sarapis, douze ans après la première, en 146/5, précisément l'année où sont enregistrées des offrandes faites sous son sacerdoce. Le passage du démotique à l'ethnique pour des clérouques ayant

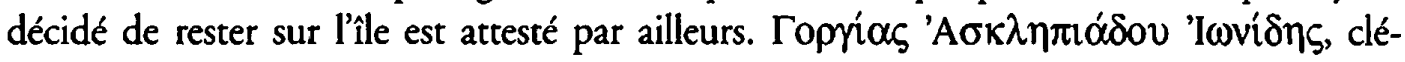
rouque (ID 1979), prêtre (ID 1499), agoranome (ID 1500), gymnasiarque (ID 1504,

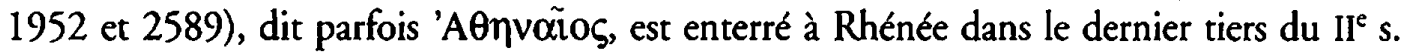
$\left(\text { Stèles, } \mathrm{n}^{\circ} 79\right)^{29}$. 
Je considère que l'on peut désormais dater les deux prêtrises de $\Phi$ i $\lambda$ okpótns

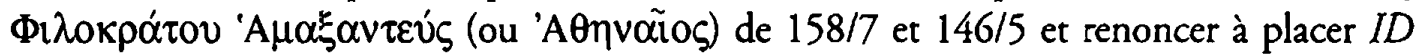
2068-2069 après 110/09, mais en 146/5 (comme peut-être d'ailleurs ID 2114) (cf. infra pour quelques remarques sur les prêtrises datées entre $166 / 5$ et 140/39).

Demeure enfin le problème posé par $I D 2133$ qui nous livre le démotique d'un

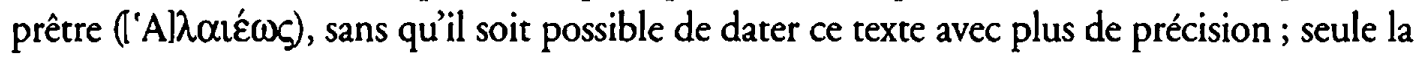
mention d'Harpocrate, invoqué en quatrième position après Sarapis, Isis et Anubis, pourrait être l'indice d'une date assez récente (la première mention datée d'Harpocrate au Sarapieion $C$ date de 127/6, ID 2146), quoique Harpocrate soit présent au Sarapieion B dès avant $166(I G \mathrm{XI} 4,1260)$ et au Sarapieion $\mathrm{A}$ très peu de temps après cette date $(I D 2116$ et 2135). Si l'on devait placer toutefois ce sacerdoce après 110/09, la date de 108/7 (plutôt que $106 / 5$ ou 94/3) est celle qui conviendrait le mieux pour ce prêtre issu de la deuxième tribu.

Quant au nom du prêtre mentionné dans le fragment $I D 2214$, les traces de lettres qui en subsistent ne permettent pas de l'identifier à l'un des personnages déjà connus.

Résumons-nous: il semble plus que probable que la rotation des tribus selon l'ordre officiel se soit poursuivie après 110/09 (malgré le "faux départ " de 109/8-108/7 qu'il n'est pas possible d'expliquer) et ce jusqu'aux dévastations des hommes de Mithridate en 88. Les raisons pour lesquelles $P$. Roussel voulait placer au moins deux prêtres après 88/7 ne s'imposent pas; en fait, il apparaît qu'aucun prêtre n'est vraisemblablement mentionné après la date fatidique de $88 / 7$; le temple a dû être saccagé par les troupes pontiques et l'utilité d'un prêtre n'apparut plus à qui que ce soit. C'est à un zacore, peut-être bien connu pour avoir exercé cet office au moins une ou deux fois auparavant, que dut revenir la charge de s'occuper de ce qui restait du sanctuaire ; ses années de zacorat servent alors à dater les dédicaces, dont deux seulement nous sont parvenues pour cette époque. Quant à la double prêtrise de Philocratès, fils de Philocratès, elle est à placer en 158/7 et $146 / 5$ et non à deux dates postérieures à 110/09.

Au terme de cette enquête, je pense que l'on peut établir une liste des prêtres de Sarapis à Délos, pour la période allant de $109 / 8$ à $88 / 7$, qui ressemblerait à celle qui suit. Les prêtrises datées avec certitude sont en caractères gras, celles datées avec de très fortes présomptions sont en caractères fins, les prêtrises datées avec plus ou moins d'incertitude sont en caractères italiques.

'A

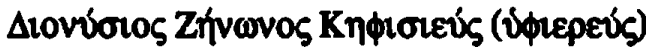

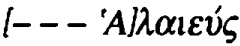

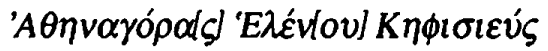




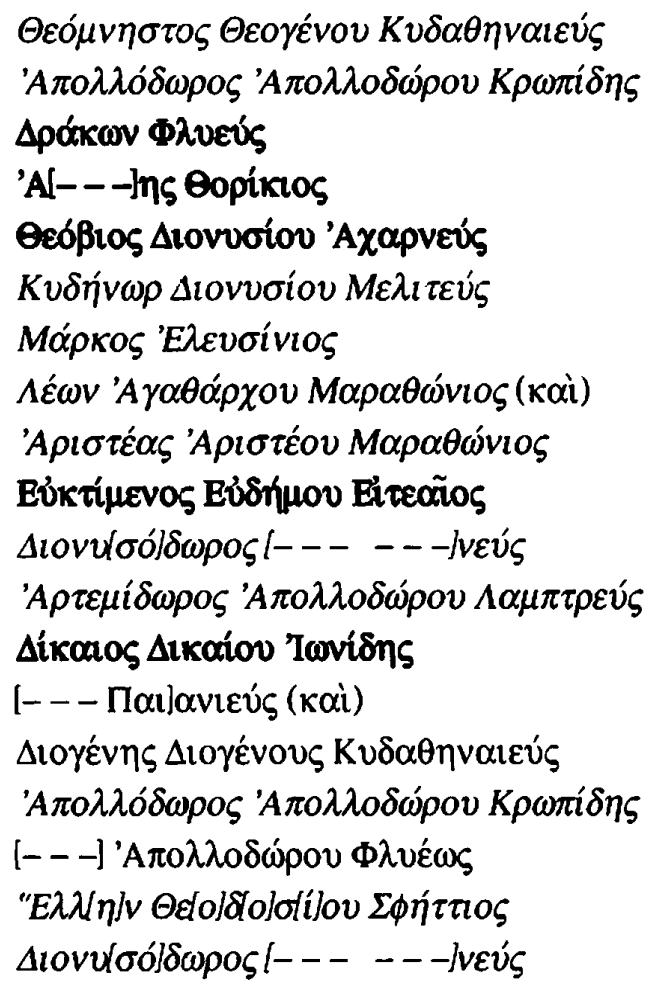

$\begin{array}{ll}(105 / 4) & \text { III } \\ (104 / 3 \text { ou } 92 / 1) & \text { IV } \\ (103 / 102) & \text { V } \\ (102 / 1) & \text { VI } \\ (101 / 0) & \text { VII } \\ (100 / 99) & \text { VIII } \\ (99 / 8) & \text { IX } \\ & \\ (98 / 7) & \text { X } \\ (97 / 6) & \text { XI } \\ (96 / 5,89 / 8 \text { ou } 88 / 7) & \\ (95 / 4) & \text { I } \\ (94 / 3) & \text { II } \\ & \\ (93 / 2) & \text { III } \\ (92 / 1 \text { ou } 104 / 3) & \text { IV } \\ (91 / 0) & \text { V } \\ (90 / 89) & \text { VI } \\ (89 / 8,88 / 7 \text { ou } 96 / 5) & \end{array}$

Il resterait à tenter d'appliquer le même type d'analyse à la période 167/6-140/39. On connaît, grâce aux inventaires, les noms d'un certain nombre des prêtres ayant officié au Sarapieion C durant les 25 premières années de la colonisation athénienne. Mais l'absence de patronymique et de démotique rend, le plus souvent, la tâche quasi insurmontable en l'état. Il faudrait qu'une étude d'ensemble se porte sur les inventaires de la période athénienne pour en tirer de moins hypothétiques remarques. Voyons malgré tout ce qu'il en est.

Un relevé, que je crois exhaustif en l'état de la documentation, fait connaître les prêtres suivants pour la période 167/6-140/39.

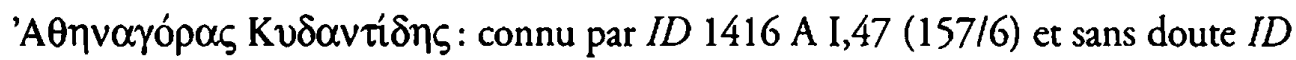
$1434 \mathrm{I}, 3,9-10,16-17(155 / 4-147 / 6)$, il appartient à la cinquième tribu $\left(P I, \mathrm{p} .7 \mathrm{n}^{\circ} 21\right)$.

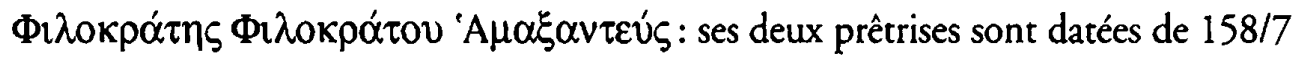
et $146 / 5$; cf. supra $\left(P I\right.$, p. 123 n $\left.^{\circ} 962,964\right)$.

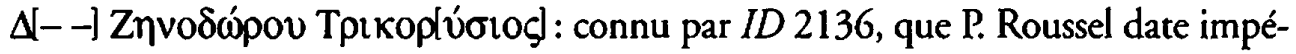
rativement d'avant $158 / 7$ sans que j'en perçoive les raisons; il appartient à la dixième tribu (PI, p. 37 n $^{\circ}$ 267).

Eipnvãios: connue par ID 1416 A I,53 (157/6), sa prêtrise est à placer vraisemblablement en 157/6, car Eipqvãios date des offrandes qui apparaissent précisément dans l'inventaire de cette année-là ( $P I$, p. 53 n $\left.^{\circ} 392\right)$. 


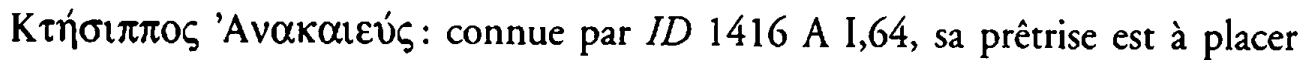
avant $157 / 6\left(P I\right.$, p. $\left.79 n^{\circ} 606\right)$; or il appartient à la neuvième tribu, tout comme Philocratès d'Hamaxantia, ce qui pose un problème. Pour respecter un ordre rigoureux de rotation des tribus, il faudrait donc supposer, soit que les deux prêtres ont exercé leur charge en même temps, ce qui me semble peu probable, soit que l'un a succédé à l'autre, ce qui est douteux. Il faut donc admettre que la rotation des tribus n'est pas absolue à cette époque. Peut-être suit-elle la chronologie des archontes athéniens. Or on sait que pour la période 160/59-142/1, elle est plus qu'incertaine.

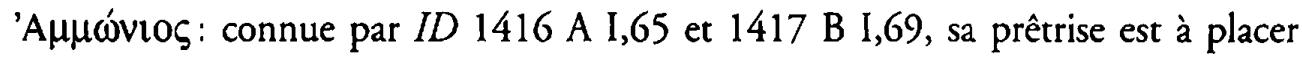
avant $157 / 6\left(P I\right.$, p. $\left.11 \mathrm{n}^{\circ} 53\right)$.

"I $\pi \pi \omega \mathrm{V}$ : connue par ID $1417 \mathrm{~A}$ II,137, sa prêtrise est à placer avant $156 / 5$ (PI, p. $\left.72 n^{\circ} 541\right)$.

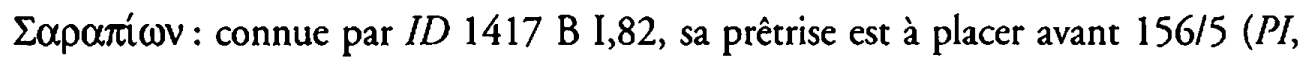
p. $\left.108 n^{\circ} 844\right)$.

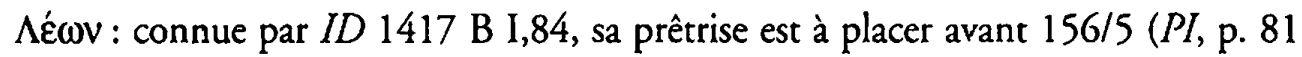
$n^{\circ}$ 626).

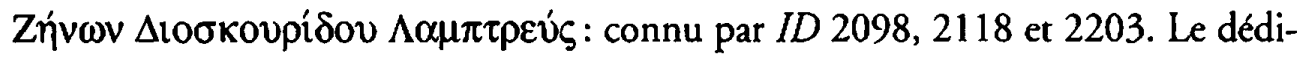
cant d'ID 2138-2139 (158/7) consacre également, sur ordre des dieux, pour sa famille, les murs d'une chapelle et l'escalier qui devait permettre d'y accéder, lors du sacerdoce de

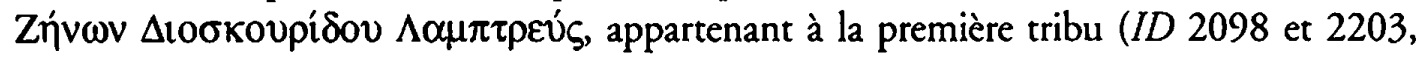
deux exemplaires, le second fort mutilé, de la même dédicace) (PI, p. $\left.63 \mathrm{n}^{\circ} 466\right)$.

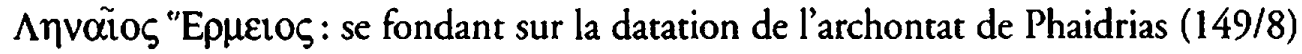
proposée par B. D. Meritt ${ }^{30}$, R. S. Fisher ${ }^{31}$ a cherché quel pouvait être le prêtre de Sarapis dans la liste de sacerdoces $I D$ 1499. S'appuyant sur la rotation officielle des tribus, il a noté

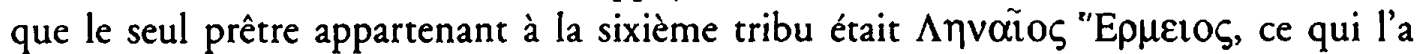
conduit à restituer le nom de ce prêtre dans $I D$ 2083. Cette hypothèse a la fragilité de son ingéniosité, d'autant que la date de 153/2 admise jusqu'alors pour l'archontat de Phaidrias semble encore solide. Le fait que l'ordre de rotation des tribus n'a visiblement pas été respecté à cette époque et que la date retenue par Fisher n'est pas certaine, loin de là, n'invite pas à retrouver assurément en $\Lambda$ ¡vỡos un prêtre de Sarapis, ni à restituer son nom dans ID 2083. Cela reste une simple possibilité (PI, p. $\left.83 \mathrm{n}^{\circ} 631\right)$.

$\Delta$ tovvo[- - ] : connue par ID 1434 I,6-7,14-15, sa prêtrise est à placer avant $146 / 5$ et peut-être plus précisément entre $156 / 5$ et $146 / 5$ (PI, p. 46 n $^{\circ} 346$ ).

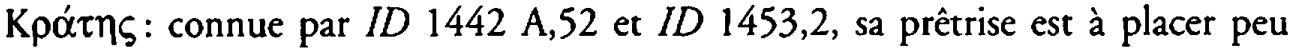
avant $145 / 4\left(P I\right.$, p. $\left.78 \mathrm{n}^{\circ} 600\right)$. 


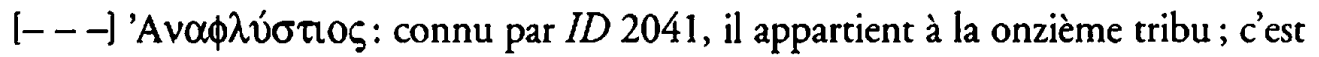
lors de sa prêtrise que le temple d'Isis fut dédié par le peuple des Athéniens.

Eủ $u \dot{v} v \eta$ : connue par ID 2140, sa prêtrise est à placer avant $140 / 39$ ou après

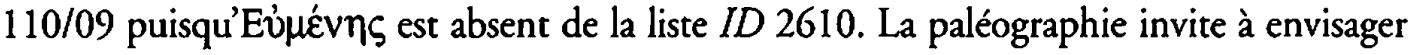
une date antérieure à $140 / 39$ ( $P I$, p. 59 n $\left.^{\circ} 438\right)$.

En partant de ces quelques observations, on doit constater qu'il est bien difficile de croire que l'ordre de rotation officiel des tribus a été suivi sans failles entre $167 / 6^{32}$ et 140/39. En admettant que le premier prêtre nommé par Athènes appartenait à la première tribu, une rotation stricte ne peut donner pour $137 / 6$ un prêtre issu de la neuvième tribu ${ }^{33}$, que l'on fixe à $167 / 6$ ou à $166 / 5$ la date de cette première nomination. De plus, on connaît deux prêtres issus de la neuvième tribu (Philocratès et Ctésippos) dont la prêtrise est impérativement à situer entre $167 / 6$ et $157 / 6$ et qui n'ont pas dû être en charge la même année, les offrandes déposées au sanctuaire sous leurs prêtrises respectives ne se suivant pas dans les inventaires qui les mentionnent.

Si l'on peut désormais tenir pour acquis que le titulaire de $158 / 7$ et de $146 / 5$ fut

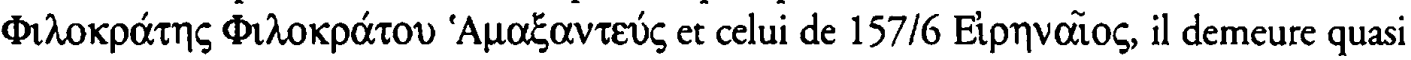
impossible de dater avec certitude les autres prêtrises. Il faut espérer que de nouveaux éléments viennent un jour nous permettre de préciser tout cela.

Pour finir, nous connaissons le nom de plusieurs des prêtres du Sarapieion C ayant exercé leur sacerdoce à l'époque de l'Indépendance, car ils sont mentionnés dans l'inventaire ID $1403 \mathrm{Bb}$ II. Composé au tout début de l'administration athénienne, il indique quelles offrandes faites au Sarapieion ont été déplacées dans l'Artémisieion (liste que l'on retrouve en partie dans l'inventaire $I D 1412$ Aa et en totalité dans $I D 1417$ A II). Cependant, de ces dix prêtres, nous ignorons presque tout. L'ordre suivi pour la présente liste, du prêtre le plus anciennement en charge au prêtre le plus récemment en charge, est celui que l'on pourrait déduire d'une étude comparée des divers inventaires, avec toure l'incertitude que cela comporte.

'Aplotóvouos: connu par ID 1403,42,61, 1412 a,[48],[56] et 1417 II,61,85 (PI, p. $\left.24 \mathrm{n}^{\circ} 160\right)$.

'ApкÉ

$\Sigma \omega \tau \varepsilon ́ \lambda \eta \varsigma$ : connu par ID 1403,48 (PI, p. $117 \mathrm{n}^{\circ} 910$ ); il apparaît, dans des séquences d'offrandes parallèles à celle d'ID 1403, dans $I D 1412$ a,50 et 1417 A II, 70 , tou-

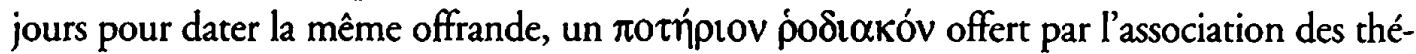

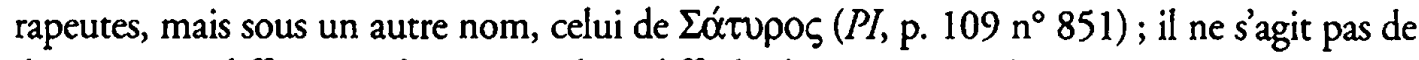
deux prêtres différents; il est cependant difficile de savoir à quel moment apparut l'erreur sur son nom : le nom fourni par ID 1403 est-il faux et un inventaire postérieur a-t-il corrigé 
cette erreur, ou bien le nom fourni par ID 1403 est-il juste et l'erreur n'est-elle apparue que dans un inventaire (de peu) postérieur, pour s'imposer ensuite? Toujours est-il qu'entre 166 et 157 une erreur ou une correction fut faite sur le nom de ce prêtre.

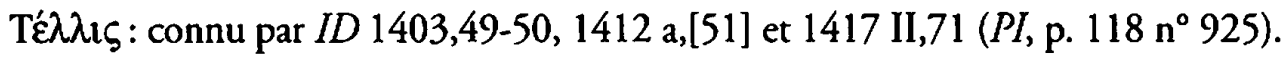

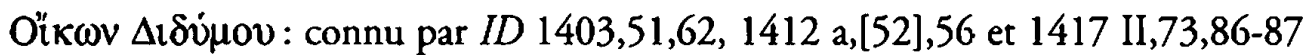
(PI, p. $\left.96 \mathrm{n}^{\circ} 741\right)$.

$\Sigma \omega \tau i \omega v \Sigma \omega \tau i \omega v o s:$ connu par ID 1403,53, 1412 a,52-53 et 1417 II,76 (PI, p. $\left.117 n^{\circ} 916\right)$.

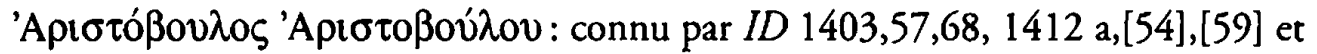
1417 II,80,93-94 (PI, p. $\left.23 \mathrm{n}^{\circ} 154\right)$.

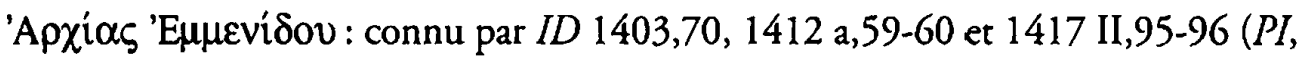
p. $\left.28 n^{\circ} 189\right)$.

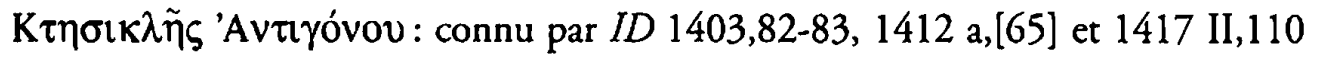
(PI, p. $\left.79 \mathrm{n}^{\circ} 605\right)$.

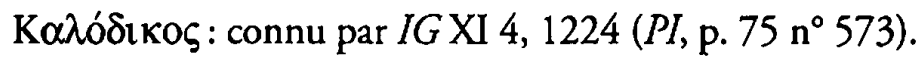

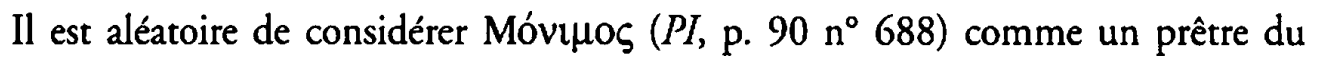
Sarapieion C à partir de la seule inscription $I G$ XI 4, 1343 (CE 46), fort mutilée, qui peut dater de la fin du III' s., et qui se présente comme suit : [O'i $\Sigma \alpha \rho \alpha$ !] $\pi \alpha \sigma \tau \alpha \mathfrak{l}$ - - - - -

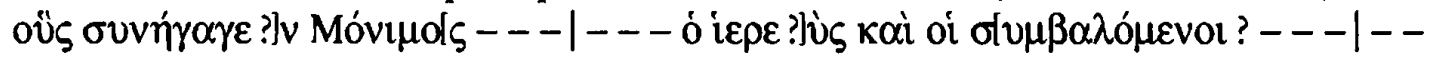

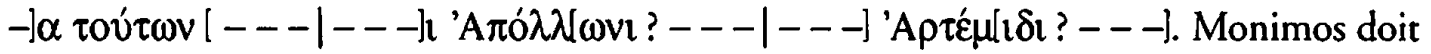
plutôt être le président d'une association de Sarapiastes tenant ses réunions dans le Sara-

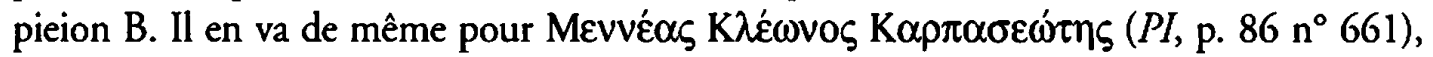
connu par plusieurs inventaires pour quatre offrandes différentes, sur une assez longue

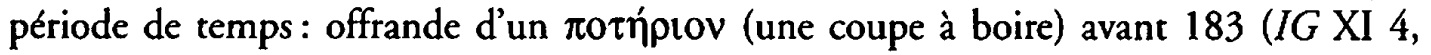
1307,8 et 1308,2-3, ID $1403 \mathrm{Bb}$ II,65, 1412 a,57 et 1417 A II,88-89), d'un $\sigma \kappa \alpha \phi i ́ o v$ (une tasse pour boire) avec l'image d'Isis et de Sarapis avant 166 (ID 1403 Bb II,72, 1412

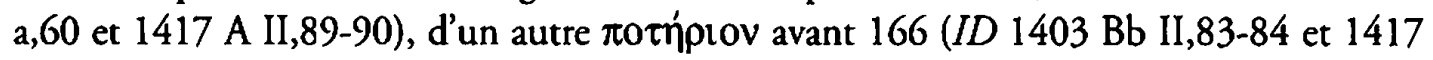

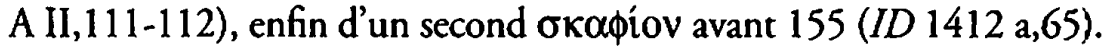




\section{Appendice}

ID 2610. Plaque de marbre blanc en deux fragments brisée en haut et à droite, qui fut remployée dans une construction. 110/09.

J'indique entre parenthèses le patronymique de chaque prêtre, qui ne figure jamais sur cette stèle, puis, dans une première colonne, la date de sa prêtrise, ensuite, dans une deuxième colonne, la concordance avec la Prosopografia Isiaca de F. Mora et enfin, dans la colonne de droite, le numéro de la tribu selon l'ordre officiel.

$[\mathrm{T}] \mu \mathrm{\mu l}---]^{34}$

$\delta \eta \mu o \sigma---1$

$\delta \eta \mu o ́ \sigma i d \varsigma]$

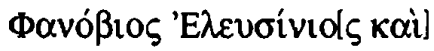

$5 \Delta \eta \mu \dot{\tau} \tau \rho \mathrm{s}$

$(137 / 6)$

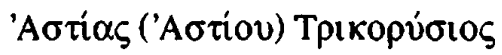

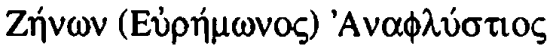

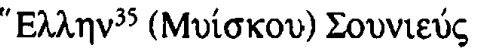

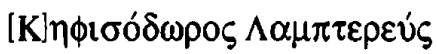

$10[\mathrm{M}] \varepsilon \dot{v} \alpha v \delta \rho \circ \varsigma \Phi 1 \lambda \alpha \dot{\delta} \eta \varsigma$

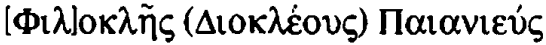

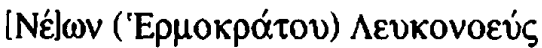

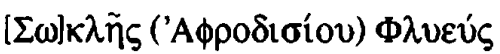

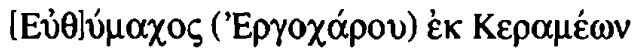

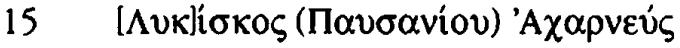

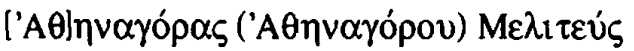

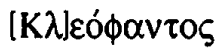

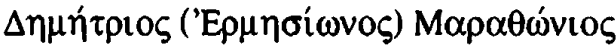

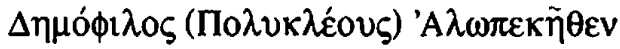

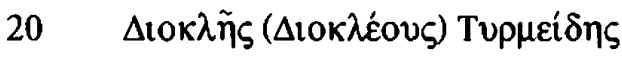

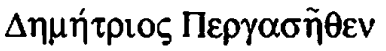

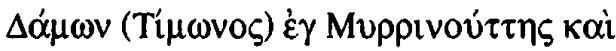

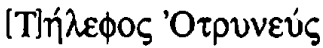

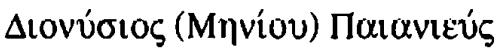

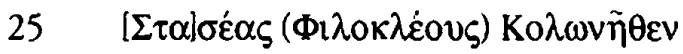

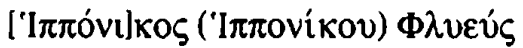

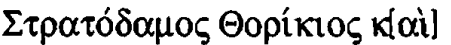

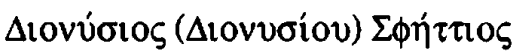

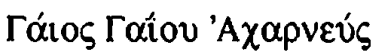

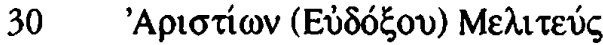

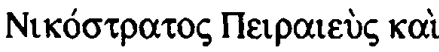

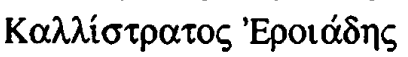

$(135 / 134)$

$(132 / 1)$

$(130 / 29)$

(129/8)

$(127 / 6)$

$(126 / 5)$

(125/4)

$(121 / 0)$

$(120 / 19)$

$(119 / 8)$

$(118 / 7)$

(117/6)

$(116 / 5)$

$(114 / 3)$

$(113 / 2)$ p. $133 n^{\circ} 1079$

p. $121 \mathrm{n}^{\circ} 944$ IX

p. $40 n^{\circ} 288$

p. $30 n^{\circ} 205 \quad X$

p. $63 n^{\circ} 466 \quad X I$

p. $54 n^{\circ} 405 \quad$ XII

p. $76 \mathrm{n}^{\circ} 581 \quad \mathrm{I}$

p. $86 \mathrm{n}^{\circ} 656$ II

p. $122 n^{\circ} 960$ III

p. $91 n^{\circ} 702$ IV

p. $115 \mathrm{n}^{\circ} 894 \quad \mathrm{~V}$

p. $58 n^{\circ} 432 \quad$ VI

p. $83 n^{\circ} 635 \quad$ VII

p. $5 \mathrm{n}^{\circ} 18 \quad$ VIII

p. $77 \mathrm{n}^{\circ} 590 \quad \mathrm{IX}(?)^{36}$

p. $42 n^{\circ} 303 \quad X$

p. $43 n^{\circ} 315 \quad X I$

p. $47 n^{\circ} 343 \quad$ XII

p. $42 \mathrm{n}^{\circ} 305$ I

p. $39 n^{\circ} 276$

p. $118 n^{\circ} 927$ II

p. $50 n^{\circ} 364$ III

p. $112 n^{\circ} 877$ IV

p. $72 n^{\circ} 540 \quad$ V

p. $113 n^{\circ} 885$

p. $48 n^{\circ} 360 \quad$ VI

p. $35 n^{\circ} 248 \quad$ VII

p. $23 n^{\circ} 152$ VIII

p. $94 \mathrm{n}^{\circ} 720$

p. $75 n^{\circ} 571$

IX 


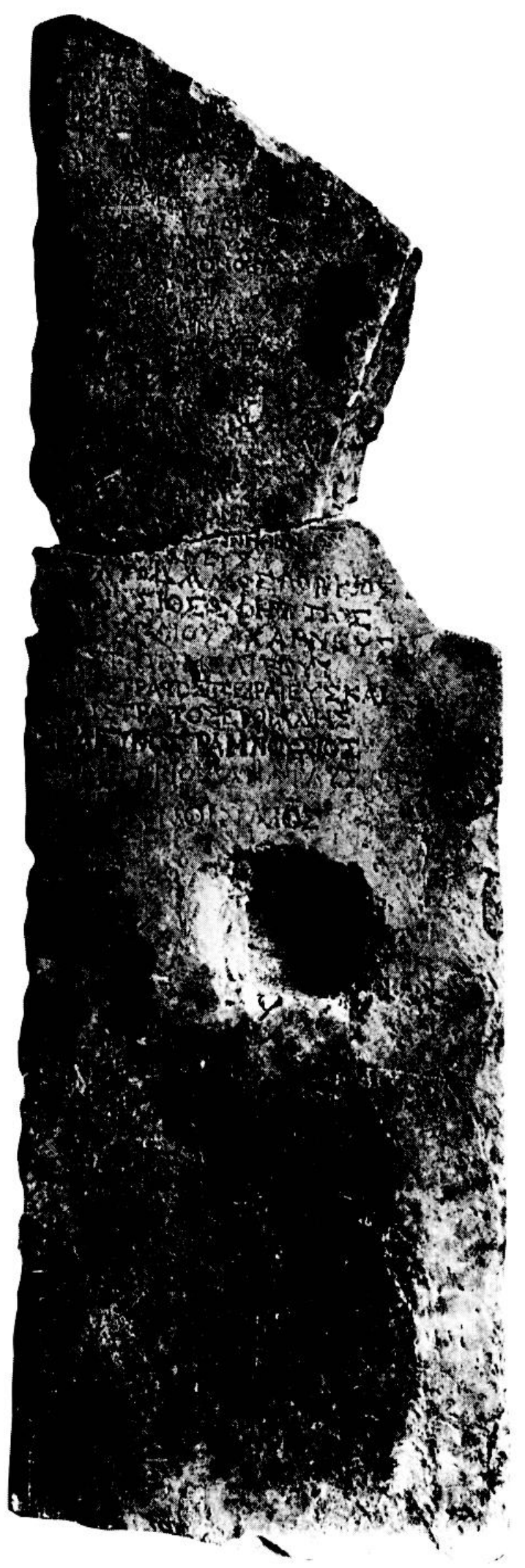

Fig. 1. 102610 (cliché EFA). 


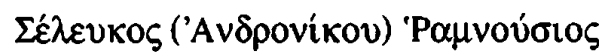

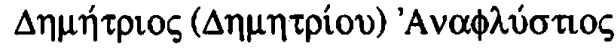 \\ 35
}

$(112 / 1)$

$\begin{array}{ll}\text { p. } 110 n^{\circ} 855 & X \\ \text { p. } 41 n^{\circ} 297 & X I \\ \text { p. } 116 n^{\circ} 907 & X I I\end{array}$

On s'est interrogé sur la personnalité et la fonction des individus mentionnés aux 1. 2-3 de cetre liste.

L. 2 A. Hauvette lisait $\triangle \mathrm{HMO}$, Th. Homolle $\triangle \mathrm{HMO \Sigma IO}$ (en s'inspirant sans doute trop de la 1.3 car il me semble impossible que l'on ait pu lire sur cette ligne plus de cinq lettres), P. Roussel $\triangle$ HMOU. Comme l'écrivait fort justement ce dernier à partir de sa lecture : "la l. 3 peut justifier la restitution $\left.\Delta \eta \mu o ́ l \sigma \iota_{0}\right]^{37}$, mais les vestiges qui subsistent ne garantissent ni n'infirment cette restitution" ( $C E$, p. 123). On ne pouvait donc jusqu'alors considérer avec certitude

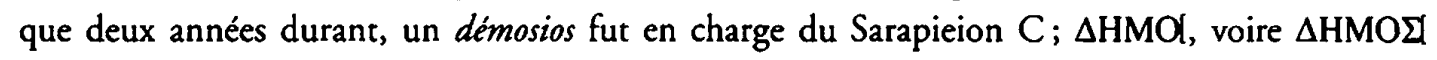
pouvaient n'être que le début d'un nom comme e.g. $\Delta \eta \mu 0 \sigma \theta \varepsilon ́ v \eta$ s. Cependant, une révision de la pierre en mai 1996 m'a permis de repérer, après le $\Sigma$, la partie inférieure d'une haste verticale - à moins qu'il ne s'agisse simplement d'un éclat ou d'une éraflure, mais je ne le pense pas - qui appartiendrait évidemment plutôt à un I qu'à un $\Theta$. Dans ce cas, si ma lecture est exacte, la restitution $\delta \eta \mu o ́ \sigma(o g)$ semble s'imposer; comme le notait déjà W. S. Ferguson, ce nom ne peut guère être qu'un substantif.

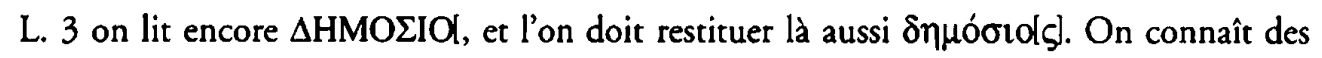

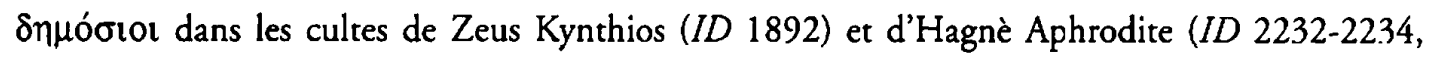
2255-2256), mais il est bien difficile de préciser leur rôle exact. Il semble en fait qu'Athènes ait confié au moins un an la responsabilité du sanctuaire à l'un de ses esclaves publics, sans doute à cause d'un problème interne à la cité attique dont nous ne savons rien. 
*Abréviations utilisées:

$C E=P$. Roussel, Cultes égyptiens (1916)

$P I=$ F. MORA, Prosopografia Isiaca, EPRO $113(1990)$.
1 Le fragment inférieur (I. 25-35) fut publié par A. HauvetTe, BCH 6 (1882), p. $350 n^{\circ}$

78 et les deux fragments réunis par Th. HOMOLLE, BCH 17 (1893), p. 146 ; $P$. ROUSSEL en donna une nouvelle édition dans ses $C E$, sous le $n^{\circ} 73$ et fig. 16. Je redonne ce texte avec une photographie et les correspondances avec $P I$, en appendice à la fin de cette étude. Toutes les dates s'entendent av. J.-C.

2 S. V. TRACY, Contributors of First Fruits for the Pythaiis, Beiträge zur klassichen Philologie 139 (1982).

3 W. B. DINSMOOR, The Atherian Archon List in the Light of Recent Discoveries (1939), p. 288-289.

4 M. GUARDUCCI, "Antichità greche nel Museo di Treviso ", ASAA 30-32 (19521954), p. 175-184 et fig. 1.

5 Pour les archontes athéniens de l'époque hellénistique, of. la liste proposée par B. D. MERIT, "Athenian Archons 347/6. 48/7 B.C.", Historia 26 (1977), p. 161-191.

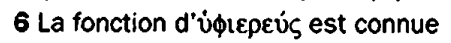
également par une inscription de Tégée IIG V 2, 49,4).

7 La découverte de ce texte permit a M. Guarducci de compléter autant que faire se put ID 2065 et de rejeter au passage les restitutions proposées par P. Roussel pour les I. 1 et 5 (CE 144 suivi par ID 2065).

8 Le stemma de cette famille peut se présenter ainsi (cf. ID 1885):

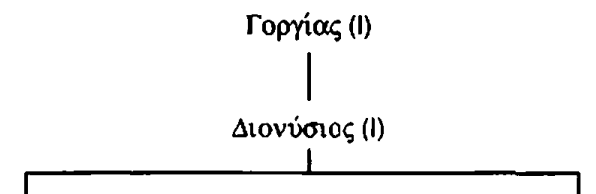

Zńvov (I)

prêtre des dieux du Cynthe 103/2

(IG II/III 2, 2336,50)
(ID $2065+$ inscr. Trévise)

\begin{tabular}{|c|c|c|}
\hline Zńvwv (II) & 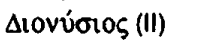 & Гoprís (II) \\
\hline ID 1884-1885, 1982 & ن́фıєрعús de & $(101885)$ \\
\hline cleidouque des & Sarapis & \\
\hline dieux syriens & $109 / 8$ & \\
\hline $107 / 6$ & (1D $2065+$ inscr. & \\
\hline (ID 2232, 2249) & Trévise) & \\
\hline $\begin{array}{l}\text { pompostole } \\
\text { (10 2608) }\end{array}$ & $(I D 1885,1982)$ & \\
\hline
\end{tabular}

9 Une conclusion qu'elle réaffirme dans ASAA 37-38 (1959-1960), p. 429.

10 Par exemple F. DUNAND, Le culte d'Isis dans le bassin oriental de la Méditerranée. EPRO 26/III (1973), dans sa

Prosopographie du clergé isiaque ou encore dans PI, où F. Mora s'inspire toutefois, de manière critique, des propositions de M. Guarducci.

11 D'après la finale de son démotique. Dionysodoros peut appartenir à l'un des

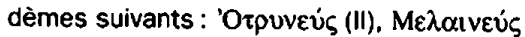

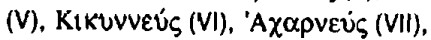

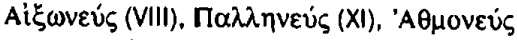
ou 'Atnveús (XII).

12 La publication de la stèle de Trévise a montré que la restitution du nom de ce prêtre dans ID 2065, proposée par P. Roussel, était caduque.

13 Sur les épimélètes de Délos, cf. la liste dressée par Chr. HABICHT, "Zu den Epimeleten von Delos 167/88 *, Hermes 119 \{1991\}, p. 194-216, qui réactualise celle dressée par P. Roussel dans Délos, colonie athénienne (1916), p. 100-118. 14 Et peutêtre 2039 et 2159.

15 W. B. DINSMOOR, op. cit. (supra, n. 3), p. $288-289$.

16 Cela pourrait confirmer la première restitution (longue) de $P$. Roussel pour

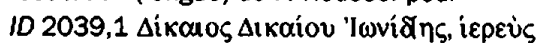

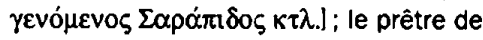
Sarapis de $94 / 3$, sorti de charge, est honoré lors de l'épimélétat d"A Aoros

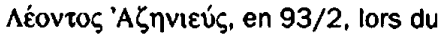

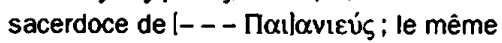
prêtre doit être mentionné dans ID 2159

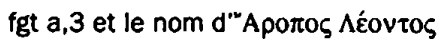

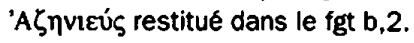
17 II faut alors restituer le nom d'A Аолос

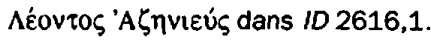
$18 \mathrm{P}$. Roussel juge improbable qu'il s'agisse ici du patronymique et du démotique du prêtre de Sarapis, mais plutôt de celui du second surintendant aux affaires sacrées, qui aurait remplacé ou aurait été remplacé

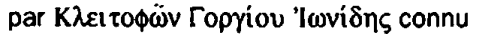

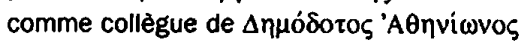

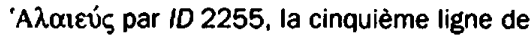
l'inscription étant trop courte pour renfermer le nom du prêtre ; comme seule la partie droite du bloc est conservée, il est difficile de préciser la longueur des lignes, d'autant qu'en début dc I. 3 on doit vraisemblablement restituer le nom d'Anubis, voire celui d'Harpocrate, créant ainsi une longueur de ligne suffisante pour restituer l'intégralité du nom d'un prêtre dont l'absence étonnerait pour une dédicace faite par le peuple des Athéniens dans l'enceinte du Sarapieion C. 19 Cette datation quasi assurée donne la date d'10 2255 qui fait mention de l'épimélète $\Delta \eta \mu \dot{\varepsilon} \alpha c$. 
20 La stèle funéraire d'un "E $\lambda \lambda\lceil\eta \mid v$

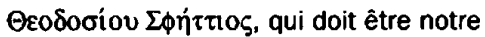
homme, est connue à Athènes (IG II/III 2 , 7516). II n'est cependant pas possible en l'état de la dater avec précision.

21 Et non pas de Ptolémée VII (M.Fr. BASLEZ, Recherches sur les conditions de penetration et de diffusion des religions orientales à Délos [1977], p. 317), ni de Ptolémée VIII (PI, p. 85, 106).

22 M.Fr. BASLEz, Délos (1977), p. 191. 23 Et pas seulement lors de son exil chypriote, de 107/6 à 89/8. En effet, dans ID 1531, datée avec certitude de 111/0, Ptolémée Sôter II se fait déjà appeler

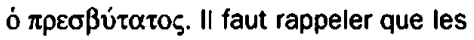
tensions entre Cléopâtre III et Sôter II apparurent rapidement lors de leur règne conjoint; avant d'être évincé pour plusieurs années à partir de $107 / 6$ au profit de son jeune frère, Sôter II avait déjà été écarté du trône au moins à deux reprises par sa mère (cf. P. W. PESTMAN, Chronologie égyptienne d'après les textes démotiques, Papyrologica Lugduno-Batava XV [1967], p. 66-68). II se fit sans doute appeler ó $\pi \rho \varepsilon \sigma \beta u ́ \tau \alpha \tau o \zeta$ dès qu'il se sentit en difficulté face à son cadet. en $111 / 0$ et peut-être même avant.

24 Certes, on pourrait supposer que la dédicace est faite pour un souverain légitime, l'aîné, enfin de retour sur un trône qui est le sien, suite à une aide (financière) apportée par celui-ci pour la restauration d'un temple sérieusement endommagé (ce qui est au moins vraisemblable) par les troupes de Mithridate en 88. La dédicace à Ammon est moins compréhensible, sauf à suivre l'idée de M.Fr. Baslez, ou d'estimer que le prêtre s'adresse à Ammon parce que Sarapis, Isis et les autres ont abandonné le sanctuaire, puisqu'ils n'ont pas empêché sa destruction partielle. Cela me semble pour le moins improbable.

25 Ce qui est vraisemblable ; la canéphore de $\Lambda \varepsilon ́ \omega v$ n'est pas nommée dans les textes. 26 Le Titanic n'a pas attendu trente ans pour couler.

27 Le problème de l'utilisation de l'ethnique athènien ou du dèmotique a ètè discuté à plusieurs reprises par M.Fr. Baslez, dans les Acts of the 8th International Congress for Greek and Latin Epigraphy II (1987), p. 46-50, dans The Greek Renaissance in the Roman Empire (1989), p. 17.36 et dans L'étranger dans le monde grec II (1991), notamment p. 25-26.

28 Proche est le cas d'un père portant le

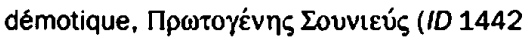

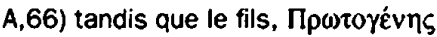

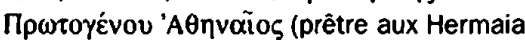

entre 135 et 105 [ID 2595], et mort à Délos au ler s. [Stèles, $n^{\circ} 459$; cf. PI, p. $105 n^{\circ} 810$ ]) porte l'ethnique, dans cette famille bien installée à Délos.

Cf. P. ROUSSEL, op. cit. (supra, n. 13), p. 59 $n^{\circ} 8$.

29 Cf. Pl, p. 36-37 n० 262.

30 B. D. MERIT, loc. cit. (supra, n. 5), p. 184. Elle n'est pas acceptée par tous, puisque Chr. HABICHT, loc. cit. (supra, n. 13), p. 197, par exemple, fait toujours de

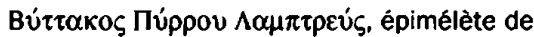
l'ile sous Phaidrias, celui de 153/2 ; cf. déjà, id., Hesperia 57 (1988), p. 240 et n. 27 ; voir également G. BUGH, ZPE 83 (1990), p. 25-35.

31 R. S. FISHER, “ A New Priest of Sarapis on Delos ", ZPE 58 (1985), p. 117-118.

32 À propos de la difficulté à fixer l'année durant laquelle furent nommés les premiers fonctionnaires athéniens sur Délos (167/166 ou 166/165), Chr. Habicht m'écrit (lettre du 2 décembre 1996): "Dieselbe Ungewißheit bestand lange für den Beginn der Liste der Gymnasiarchen (ID 2589) : $167 / 6$ nach Roussel, $166 / 5$ nach Plassart. Die noch immer unveröffentlichte Inschrift zu Ehren des Gymnasiarchen Pausanias von Melite (RA 1948 I 263-4) zeigt, daß dieser Gymnasiarch im Jahr des athenischen Archons Pyrrhos (158/7) war ; ID 2589 zeigt inn als zehnten Inhaber des Amtes, so daß der erste Gymnasiarch 167/6 amtiert haben muß (vermutlich nur einen Teil des attischen Jahres). Dies dürfte dafür sprechen, daß auch der erste athenische Sarapispriester in Delos sein Amt schon 167/6 angetreten hat, nicht erst 166/5 *.

33 Même en comptabilisant (ou en

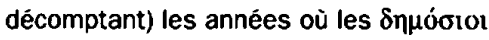
furent en charge du sanctuaire.

34 Une révision de la pierre en mai 1996 $m$ 'a permis de repérer après le $M$ la partie inférieure d'une haste oblique qui pourrait bien appartenir à un A; cependant les noms commençant par TIMA- sont trop nombreux pour permettre une proposition solide. 35 L'E initial se distingue sur la pierre (lecture de mai 1996).

36 Malgré l'absence de démotique, il serait logique que ce prêtre appartînt à la neuvième tribu.

37 La position du premier crochet dans cette citation, sauf erreur typographique, peut poser le problème de savoir si P. Roussel a bien lu $\triangle H M O य$ comme il l'écrit p. 124 ou simplement $\triangle H M O$; je pencherais plutôt pour l'erreur typographique. 